\title{
Continuous Markov Equilibria with Quasi-Geometric Discounting $^{1}$
}

\author{
Satyajit Chatterjee and Burcu Eyigungor \\ Federal Reserve Bank of Philadelphia
}

February 11, 2014

\footnotetext{
${ }^{1}$ Corresponding Author: Satyajit Chatterjee, Research Department, Federal Reserve Bank of Philadelphia, Ten Independence Mall, Philadelphia PA, 19106; (215) 574-3861, satyajit.chatterjee@phil.frb.org. The authors thank Marina Azzimonti, Roc Armenter, Per Krusell and seminar participants at the IIES Stockholm, FRB of Philadelphia and FRB Minneapolis for helpful comments. The views expressed here are those of the authors and do not necessarily represent the views of the Federal Reserve Bank of Philadelphia or the Federal Reserve System.
} 


\begin{abstract}
We prove that the standard quasi-geometric discounting model used in dynamic consumer theory and political economics does not possess continuous Markov Perfect equilibria if there is a strictly positive lower bound on wealth. We also show that at points of discontinuity, the decision maker strictly prefers lotteries over next period's assets. We then extend the standard model to have lotteries and establish the existence of a MPE with continuous decision rules. The model with and without lotteries are numerically compared and it is shown that the model with lotteries behaves more in accord with economic intuition.
\end{abstract}

Keywords: Quasi-geometric, Quasi-hyperbolic, Markov Perfect Equilibrium, Debt Limit, Continuous Solutions, Lotteries

JEL Codes: D11, E62 


\section{Introduction}

Intertemporal preferences with quasi-geometric (or quasi-hyperbolic) discounting has been proposed for studying optimal national savings policy in overlapping generations models with imperfect altruism (Phelps and Pollak (1968)), for studying the savings behavior of households in a situation where individuals value commitment to a consumption plan (Laibson (1997)), and for studying optimal growth outside of the strait-jacket of geometric discounting (Barro (1999)).

Quasi-geometric discounting also arises endogenously in environments that feature collective decision making by entities that discount geometrically at a common rate but do not value each others consumption equally. Examples include models of political turnover and disagreement (Alesina and Tabellini (1990) and Persson and Svensson (1989)), models of majority-based legislative decision making (Battagliani and Coate (2008)), and nonunitary models of household decision making (Hertzberg (2012)) $!^{1}$

This two-pronged appeal of quasi-geometric discounting motivates our paper. To explain our contributions, consider a decision maker (henceforth DM) with an infinite planning horizon, constant endowment, and facing a constant interest rate $r$. Suppose that (the long run) discount factor between any two future consecutive periods is $\beta$, while the discount factor between the current and next period is $\delta \beta$, where $\delta<1$. In addition, $(1+r) \beta=1$. When attention is focused on the Markov perfect equilibria of this model, the expectation is that the present bias induced by $\delta$ will be a force in favor of dissaving and cause the DM to behave as if it is impatient. Indeed in cases where an analytical solution exists, this expectation is confirmed and the MPE decision rule displays smooth dissaving behavior that is observationally equivalent to a geometric discounting model with a $\beta<1 /(1+r)$ ? $^{2}$

But when an equilibrium of this model is computed via standard value function iteration

\footnotetext{
${ }^{1}$ Several recent political economics models (Acemoglu, Golosov, and Tsyvinski (2011), Aguiar and Amador (2011), and Azzimonti (2011)) feature some version of present bias.

${ }^{2}$ Of course, the two models have very different implications with regard to the value of commitment to a consumption path for the DM.
} 
methods, the decision rule found is neither continuous nor does it necessarily display dissaving behavior everywhere. Instead, the decision rule typically displays multiple steady states and the DM dissaves until a steady state is reached and then the dissaving behavior stops. This occurs even in the model for which a smooth analytical solution with persistent dissaving is known to exist ${ }^{3}$

The simultaneous occurrence of smooth (analytical) and discontinuous (computed) solutions for the same model has generally been interpreted as a manifestation of multiple equilibria, an interpretation that seems reasonable given that Markov equilibria are generally not unique. Guided by this assessment, perhaps, researchers have been to content to restrict attention to parameter values for which discontinuous solutions ("pathologies") do not arise (Laibson, Repetto, and Tobacman (1998)) or adopted methods other than finite-state value function iteration to locate smooth equilibria (Krusell, Kuruscu, and Smith (2002), Judd (2004), Maliar and Maliar (2005)).

Our first contribution is to shed light on the source of discontinuous MPE. For the stationary environment described earlier with CRRA preferences, we show that if the DMs wealth (i.e, its present discounted value of future endowments plus current asset less current debt) cannot fall below some strictly positive value, a continuous MPE decision rule will fail to exist. To the best of our knowledge, ours is the first proof of the necessity of discontinuous MPE decision rule in the quasi-geometric discounting model. Note that the condition under which discontinuities occur is of practical interest. For instance, it applies if the DM is an individual facing a borrowing constraint or a government with finite revenue flow and minimum expenditure constraint.

The result has two immediate implications. First, the known smooth (i.e., differentiable) analytical solutions for the CRRA case work because the DM's wealth is allowed to get arbitrarily close to zero. If those models are perturbed to include an arbitrarily small but strictly

\footnotetext{
${ }^{3}$ Even in more elaborate settings, MPE decision rules with quasi-geometric discounting can feature discontinuities. For instance, Krusell and Smith (2003) give an algorithm for constructing discontinuous MPE decision rules for a model that is essentially the neoclassical growth model.
} 
positive lower bound on wealth, the smooth solution will be lost. $4^{4}$ Second, the simultaneous occurrence of a smooth analytical solution and a discontinuous computed solution is not the result of multiple equilibria. A finite-state value function iteration approach will, by design, have the same finite set of choices available to the DM at each decision node. In such a setting there is a de facto lower bound on wealth. Thus the computational approach will generate discontinuous equilibria because the model being computed is an approximation to a model with a positive lower bound on wealth and for this model, discontinuous equilibria are the only equilibria possible.

The second contribution of the paper is to propose an extension of the quasi-geometric discounting model. To motivate this extension, we show first that at any point of discontinuity of a MPE decision rule, the DM would strictly prefer to utilize lotteries over the choice of next period's assets. The reason is that a discontinuity in decision rule always reflects a non concave segment in the continuation value function. Hence, lotteries increase the welfare of the current DM.5

With this result in hand, we extend the standard quasi-geometric discounting model to have lotteries over the choice of next period's assets. The extension has several important consequences. First, any MPE decision rules for consumption and expected asset choice are now continuous functions of beginning-of-period wealth. Furthermore, consumption is now an increasing function of beginning-of-period wealth. Neither implication holds, in general, in the standard version of the model.

\footnotetext{
${ }^{4}$ The theoretical results presented in this paper pertain to the stationary environment with $(1+r) \beta=1$. It will be clear from the logic of the proof that it can be extended to encompass the case where $(1+r) \beta<1$ at the cost of some algebra. At the cost of even more algebra, it can probably be shown that our results apply if $(1+r) \beta \leq \alpha$, where $\alpha$ is some number strictly greater than 1 that is inversely related to $\delta$. The reason is that for any given degree of present bias, a $(1+r) \beta$ is substantially bigger than 1 will induce the DM to not dissave at all (or save perpetually). In this case, the lower bound on wealth is of no consequence and our proof of discontinuity will not apply.

${ }^{5}$ Since lotteries are generally equivalent to (fair) value gambles, this finding points to a hitherto unappreciated implication of quasi-geometric discounting. Behavioral justifications of quasi-geometric discounting uniformly point to the use of various commitment strategies by individuals. But individuals also willingly take risks (gamble to get out of poverty, for instance) and this, too, is consistent with quasi-geometric discounting.
} 
Second, MPE with lotteries exists under general conditions..$^{6}$ Existence proofs for the stationary environment, such as the one given in Laibson (1996), employ a guess-and-verify method that hinges on the lower bound on wealth being zero and the utility function being CRRA.7 In contrast, the proof given in this paper does not rely on guessing decision rules and works for general utility functions, for any nonnegative lower bound on wealth, and can easily be extended to the case where endowments are uncertain. We also briefly describe the algorithm we use to compute the equilibrium with lotteries.

We also numerically compare the standard quasi-geometric discounting model with its lottery-enhanced counterpart. 8 In the first set of comparisons, we simulate the model for a case where closed-form decision rules are known for the standard model. This solution implies that in the long run both consumption and wealth converge to zero. We numerically confirm that if this same model is computed with a strictly positive lower bound imposed on wealth, the computed equilibrium decision rule is discontinuous and the points of discontinuity occur at asset levels that are also (typically) steady states. We then compute the equilibrium with lotteries permitted. The computed decision rule is, of course, continuous and, interestingly, quite close to the closed-form analytical solution for wealth levels far away from the lower bound. As the wealth level approaches the lower bound, the two decision rules diverge. The debt limit associated with the lower bound on wealth is the unique steady state debt level in the model with lotteries.

In the second set, we outline how the model with lotteries can be extended to an environment with shocks to endowments. For a very similar environment with a debt limit of zero (or, equivalently, a strictly positive lower bound on wealth), Harris and Laibson (2001) prove that if the present-bias parameter $\delta$ is sufficiently close to 1 (small bias) and future

\footnotetext{
${ }^{6}$ As was shown early on by Peleg and Yaari (1973), with time-inconsistent preferences the existence of a MPE equilibrium cannot be taken for granted.

${ }^{7}$ For this set up, one can guess the unique subgame perfect Nash equilibrium strategies of the finite horizon model. The equilibrium strategy of the infinite horizon model is obtained as the point-wise limit of the (unique) finite-horizon strategies.

${ }^{8}$ It is perhaps important to note that one can compute the decision rule for expected asset choice directly, without having to keep track of the lotteries underlying this choice (the equilibrium lotteries can be inferred once the equilibrium decision rule for expected asset choice is found).
} 
endowments are drawn from a probability distribution with continuous density, MPE decision rules of the standard model are continuous in current wealth. Thus, in theory, shocks can play a role similar to lotteries. To further investigate this point, we add i.i.d. shocks to endowment and compare decision rules with and without lotteries. If $\delta$ and/or volatility of endowment shocks is high, the decision rule from the standard model is continuous and virtually identical to the decision rule from the model with lotteries. But if the volatility of shocks and/or $\delta$ is lowered (even slightly), the decision rule for the standard model can become discontinuous.

In the final set of numerical experiments, we compare the answers from the standard model and the model with lotteries for a policy question: What is the ideal debt limit from the perspective of the current DM? Many laws regulating real-world credit markets ultimately effect debt limits. The answer from the model with lotteries is intuitive: The current DM's lifetime utility with respect to the debt limit is an inverted-U. Because of the present bias, there is an ideal debt limit which allows the current DM to indulge its preference for current consumption and to which it goes immediately (starting from zero debt). For any other debt limit, utility decreases with distance from the ideal debt limit. In contrast, because equilibrium decision rules may fail to be continuous, the relationship between welfare and debt limit in the standard model may not be an inverted U. For instance, in the stationary model the ideal debt limit is not unique: there are many debt limits that deliver (approximately) the same ideal welfare level.

The paper is organized in three parts. In the first part, comprising Section 2, the standard stationary quasi-geometric discounted model is presented and analyzed. In the second part, comprising of Section 3, we extend the standard model to have lotteries. In the third part, comprising of Sections 4 through 6 , we present the numerical comparison of the model with and without lotteries. 


\section{The Standard Quasi-Geometric Discounting Model}

Here we describe a standard environment of individual intertemporal choice with quasigeometric discounting. Under some parameter restrictions, this model is isomorphic to a model of political disagreement and turnover (Alesina and Tabellini (1990)) in which two parties stochastically alternate in power with equal probability. This equivalence is shown in Appendix A.

The DM has constant stream of endowment $y$ and a period utility function $U(c)=$

$c^{1-\gamma} /(1-\gamma), \gamma>0$. The (long run) discount factor between any two future consecutive periods is $\beta<1$, while the discount factor between the current and next period is $\delta \beta$, where $\delta<1$. The DM has access to credit market where it can borrow or save at a constant interest rate. We denote the price of a unit bond or deposit by $q$ and assume that $q=\beta$. There is a lower bound on the level of assets (debt limit) $\underline{b} \leq 0$ but no upper bound. We assume that $y+(1-\beta) \underline{b}=\kappa>0$, which ensures that the DM has high enough endowment to service the maximum level of debt.

Given a continuation value function $W(b)$, the DM solves the following dynamic program:

$$
\begin{aligned}
& V(b)=\max _{b^{\prime}} U(c)+\delta \beta W\left(b^{\prime}\right) \\
& \text { s.t. } \\
& c=y+b-\beta b^{\prime} \geq 0 \text { and } b^{\prime} \geq \underline{b}
\end{aligned}
$$

Definition 1 A Markov Perfect Equilibrium (MPE) is a pair $\{a(b), W(b)\}$ such that $a(b)$ solves (1) given $W(b)$, and $W(b)$ solves:

$$
W(b)=U(y+b-\beta a(b))+\beta W(a(b)) .
$$

Definition $2 b$ is a steady state of $a M P E$ if $b=a(b)$. 


\subsection{Discontinuity of MPE Decision Rules}

The main goal of this section is to establish that any MPE decision rule $a(b)$ will fail to be continuous on the entire domain. To get to that result, we present a series of results that characterize MPE of this environment. Following the presentation of the main result (Theorem 3), some implications of the lack of continuity are also noted.

Theorem 1 In any MPE, $a(b)$ is increasing in $b$ and $a(b) \leq b$.

\section{Proof.}

Monotonicity: Suppose $b_{1}>b_{2}$. If $a\left(b_{1}\right)$ is not feasible when $b$ is $b_{2}$ then $a\left(b_{2}\right)$ must be less than $a\left(b_{1}\right)$ and we are done. Suppose then that $a\left(b_{1}\right)$ is feasible when $b$ is $b_{2}$. By optimality, $U\left(y+b_{2}-\beta a\left(b_{2}\right)\right)+\delta \beta W\left(a\left(b_{2}\right) \geq U\left(y+b_{2}-\beta a\left(b_{1}\right)\right)+\delta \beta W\left(a\left(b_{1}\right)\right)\right.$. Since $a\left(b_{2}\right)$ is clearly feasible when $b$ is $b_{1}$, we also have that $U\left(y+b_{1}-\beta a\left(b_{1}\right)\right)+\delta \beta W\left(a\left(b_{1}\right)\right) \geq$ $U\left(y+b_{1}-\beta a\left(b_{2}\right)\right)+\delta \beta W\left(a\left(b_{2}\right)\right)$. Adding up these two inequalities and re-arranging implies

$$
U\left(y+b_{1}-\beta a\left(b_{1}\right)\right)-U\left(y+b_{2}-\beta a\left(b_{1}\right)\right) \geq U\left(y+b_{1}-\beta a\left(b_{2}\right)\right)-U\left(y+b_{2}-\beta a\left(b_{2}\right)\right) .
$$

Since $b_{1}>b_{2}$ and $U$ is strictly concave, the above inequality can hold only if $a\left(b_{2}\right) \leq a\left(b_{1}\right)$.

$a(b) \leq b$ : (by contradiction). Assume that $a(b)>b$. We will show that the DM can get higher utility by choosing $b$ today when it knows that next period's choice will be $a(b)>b$. If it chooses $a(b)$ this period, it gets:

$$
U(y+b-\beta a(b))+\beta \delta W(a(b)) .
$$

If it chooses $b$ this period, it gets:

$$
U(y+b-\beta b)+\beta \delta[U(y+b-\beta a(b))+\beta W(a(b))]
$$


The difference in payoff between the deviation strategy (4) and the putative equilibrium strategy (3) is:

$$
U(y+b-\beta b)-U(y+b-\beta a(b))+\beta \delta U(y+b-\beta a(b))-\beta \delta(1-\beta) W(a(b))
$$

We will show that this difference is strictly positive when we replace $W(a(b))$ by an upper bound and, therefore, it will be true for $W(a(b))$ as well.

From today's perspective, the maximum lifetime utility the DM can get starting with $b$ tomorrow is:

$$
\widetilde{W}(b)=\max _{b^{\prime}, b^{\prime \prime}, b^{\prime \prime \prime}, \ldots} U\left(y+b-\beta b^{\prime}\right)+\beta U\left(\bar{y}+b^{\prime}-\beta b^{\prime \prime}\right)+\beta^{2} U\left(y+b^{\prime \prime}-\beta b^{\prime \prime \prime}\right) \ldots
$$

If the DM gets to choose each element of the sequence $b^{\prime}, b^{\prime \prime}, b^{\prime \prime \prime}, \ldots$ it will choose them such that consumption will be constant. Therefore

$$
\widetilde{W}(b)=\frac{U(y+(1-\beta) b)}{1-\beta} .
$$

Since $W(b)$ cannot possibly be more than $\widetilde{W}(b)$, a lower bound on the gain from deviating is obtained if we replace $W(a(b))$ in $(5)$ by $\widetilde{W}(a(b))$. This lower bound is given by:

$$
U(y+b-\beta b)-U(y+b-\beta a(b))+\beta \delta U(y+b-\beta a(b))-\beta \delta U(y+(1-\beta) a(b))
$$

Next, by the definition of $\widetilde{W}($.$) and strict concavity of U$ we have:

$$
\begin{aligned}
& \frac{U(y+b-\beta b)}{1-\beta}>U(y+b-\beta a(b))+\beta \frac{U(y+(1-\beta) a(b))}{1-\beta} \\
& \Rightarrow U(y+b-\beta b)>U(y+b-\beta a(b))+\beta[U(y+(1-\beta) a(b))-U(y+b-\beta a(b))]
\end{aligned}
$$


Replacing the first term in (7) by the r.h.s. of the above inequality, we obtain another lower bound on (5):

$$
(1-\delta) \beta[U(y+(1-\beta) a(b))-U(y+b-\beta a(b))]
$$

Since $a(b)>b$ and $(1-\delta) \beta>0,(8)$ is strictly postive. Therefore, the gain from choosing $b$ at $b$ when the policy calls for choosing $a(b)>b$ is strictly positive. Hence in any MPE $a(b) \leq b$.

Corollary $1 \underline{b}$ is a steady state of any MPE.

Since $a(b) \leq b$ implies $c(b) \geq y+(1-\beta) b$, recalling the definition of $\kappa$ gives a second useful corollary:

Corollary 2 In any $M P E, c(b) \geq \kappa>0$.

The next result states that there is (right) neighborhood of $\underline{b}$ such that if the DM starts with $b$ in that neighborhood, its optimal choice is to go to the debt limit. Define $b_{1 M}$ to be such that $U^{\prime}\left(y+b_{1 M}-\beta \underline{b}\right)=\delta U(y+(1-\beta) \underline{b})$. Since $U^{\prime}$ is decreasing and $\delta<1$, it is evident that $b_{1 M}>\underline{b}$.

Theorem 2 In any $M P E, a(b)=\underline{b}$ for $b \in\left(\underline{b}, b_{1 M}\right]$.

Proof. We will show that for $b \in\left(\underline{b}, b_{1 M}\right]$, choosing $\underline{b}$ gives strictly higher utility than any other feasible choice of $b^{\prime}$. Define the equilibrium payoff from choosing $b^{\prime}$, given $b$, as:

$$
\Phi\left(b, b^{\prime}\right)=U\left(y+b-\beta b^{\prime}\right)+\delta \beta W\left(b^{\prime}\right) .
$$

and define

$$
\widetilde{\Phi}\left(b, b^{\prime}\right)=U\left(y+b-\beta b^{\prime}\right)+\delta \beta \widetilde{W}\left(b^{\prime}\right) .
$$


Since $\widetilde{W}(b) \geq W(b)$, it follows that

$$
\widetilde{\Phi}\left(b, b^{\prime}\right) \geq \Phi\left(b, b^{\prime}\right)
$$

Next, consider the program:

$$
\max _{b^{\prime} \geq \underline{b}} \widetilde{\Phi}\left(b, b^{\prime}\right)=\max _{b^{\prime} \geq \underline{b}}\left\{U\left(y+b-\beta b^{\prime}\right)+\delta \beta\left[\frac{U\left(y+(1-\beta) b^{\prime}\right)}{(1-\beta)}\right]\right\}
$$

where we have replaced $\widetilde{W}(b)$ by the equivalent expression in $(6)$. The FOC for the optimal choice of $b^{\prime}$ is

$$
-U^{\prime}\left(y+b-\beta b^{\prime}\right)+\delta U^{\prime}\left(y+(1-\beta) b^{\prime}\right) \leq 0
$$

For $b^{\prime}=\underline{b}$, this condition holds with an equality when $b=b_{1 M}$ (this follows from the definition of $\left.b_{1 M}\right)$ and it holds with a strict inequality for when $b \in\left[\underline{b}, b_{1 M}\right)$. Then, from the strict concavity of $U$ it follows

$$
\widetilde{\Phi}(b, \underline{b})>\widetilde{\Phi}\left(b, b^{\prime}\right) \text { for } b \in\left(\underline{b}, b_{1 M}\right] \text { and } b^{\prime}>\underline{b} .
$$

Next, by Corollary 1 we know that in any MPE $a(\underline{b})=\underline{b}$ and, therefore, $W(\underline{b})=\widetilde{W}(\underline{b})$. Hence,

$$
\Phi(b, \underline{b})=\widetilde{\Phi}(b, \underline{b}) .
$$

Finally, combining (10), (11) and $(12)$ gives:

$$
\Phi(b, \underline{b})=\widetilde{\Phi}(b, \underline{b})>\widetilde{\Phi}\left(b, b^{\prime}\right) \geq \Phi\left(b, b^{\prime}\right) \text { for } b \in\left(\underline{b}, b_{1 M}\right] \text { and } b^{\prime}>\underline{b} .
$$

Thus, for $b \in\left(\underline{b}, b_{1 M}\right]$, choosing $\underline{b}$ gives strictly higher utility than any other feasible choice regardless of $W(b)$. Hence $a(b)=\underline{b}$ for $b \in\left(\underline{b}, b_{1 M}\right]$. 
The next result establishes that if $a(b)$ is assumed to be continuous, there is a (right) neighborhood of $b_{1 M}$ for which the value of $a(b)$ is pinned down. Define $b_{2 M}$ to be such that $U^{\prime}\left(y+b_{2 M}-\beta b_{1 M}\right)=\delta^{2} U^{\prime}\left(y+b_{1 M}-\beta \underline{b}\right)$. From the definition of $b_{1 M}$ (and that $U^{\prime}$ is decreasing and $\delta<1$ ), we may verify that $b_{2 M}>b_{1 M}$. Then we have:

Lemma 1 In any MPE with continuous $a(b)$,

$$
a(b)=\left\{\begin{array}{cc}
\underline{b} & b \in\left[\underline{b}, b_{1 M}\right] \\
\frac{\left(-\left(1-\delta^{1 / \gamma}\right) y+\delta^{1 / \gamma} b+\beta \underline{b}\right)}{1+\delta^{1 / \gamma} \beta} & b \in\left(b_{1 M}, b_{2 M}\right] .
\end{array}\right.
$$

Proof. By Theorem 1 and $2, a(b)=\underline{b}$ for $b \in\left[\underline{b}, b_{1 M}\right]$. To prove the rest, we will guess that when $b \in\left(b_{1 M}, b_{2 M}\right)$ the optimal choice of $b^{\prime}$ is obtained from a maximization problem in which the DM optimally chooses $b^{\prime}$ from the set $\left[\underline{b}, b_{1 M}\right]$. By Theorem 1 and 2 , the DM can predict that for any $b^{\prime}$ in this set, future DMs will choose $\underline{b}$. Therefore, the guess, which we denote as $h(b)$, solves:

$$
h(b)=\underset{b^{\prime}}{\operatorname{argmax}}\left\{U\left(y+b-\beta b^{\prime}\right)+\delta\left[\beta U\left(y+b^{\prime}-\beta \underline{b}\right)+\beta^{2} \frac{U(y+(1-\beta) \underline{b})}{(1-\beta)}\right]\right\}
$$

s.t.

$\underline{b} \leq b^{\prime} \leq b_{1 M}$

Since (13) is a strictly concave problem with convex constraint set, $h(b)$ exists and the FOC for $b^{\prime}$ is both necessary and sufficient to characterize $h(b)$. Taking account of corner solutions, the FOC is:

$$
-U^{\prime}\left(y+b-\beta b^{\prime}\right)+\delta U^{\prime}\left(y+b^{\prime}-\beta \underline{b}\right) \begin{cases}\leq 0 & \text { if } b^{\prime}=\underline{b} \\ =0 & \text { if } \underline{b}<b^{\prime}<b_{1 M} \\ \geq 0 & \text { if } b^{\prime}=b_{1 M}\end{cases}
$$


Using the definitions of $b_{1 M}$ and $b_{2 M}$, we may verify that for $b$ in the open interval $\left(b_{1 M}, b_{2 M}\right)$, the FOC is satisfied with equality. The parametric form for $U$ then gives:

$$
h(b)=\frac{\left(-\left(1-\delta^{1 / \gamma}\right) y+\delta^{1 / \gamma} b+\beta \underline{b}\right)}{1+\delta^{1 / \gamma} \beta}, b \in\left(b_{1 M}, b_{2 M}\right) .
$$

Note that the postulated $a(b)$ is consistent with the maintained assumption that $a(b)$ is continuous in $b$, since $\lim b_{\downarrow} b_{1 M} h(b)=\underline{b}$ (the quickest way to see this is to observe that the condition that defined $b_{1 M}$ is the same as the FOC from which $h(b)$ is derived). Note also that $h(b)$ strictly increasing in $b \in\left(b_{1 M}, b_{2 M}\right)$ with $\lim _{b \uparrow b_{2 M}} h(b)=h\left(b_{2 M}\right)=b_{1 M}$. Hence the guess is also consistent with Theorem 1 .

Next we will verify that our guess is the only solution for $b \in\left(b_{1 M}, b_{2 M}\right]$ if $a(b)$ is to be continuous. For this, it is sufficient to verify that $h(b)$ is the correct guess for $b \in\left(b_{1 M}, b_{2 M}\right)$ : if $a(b)$ is indeed equal to $h(b)$ over the open interval then by the assumed continuity of $a(b)$, $a\left(b_{2 M}\right)$ must equal $\lim _{b \uparrow b_{2 M}} h(b)=h\left(b_{2 M}\right)=b_{1 M}$.

To proceed, suppose there is $\hat{b} \in\left(b_{1 M}, b_{2 M}\right)$ for which $a(\hat{b}) \neq h(\hat{b})$. Two cases are possible. (i) Suppose that $\underline{b} \leq a(\hat{b}) \leq b_{1 M}$. Then we know that the DM must believe that all future asset choices must be $\underline{b}$ and in that case we know from $(13)$ that $h(\hat{b})$ is the optimal choice. Since $a(\hat{b}) \neq h(\hat{b})$, the supposition (i) must be false. (ii) Suppose that $a(\hat{b})>b_{1 M}$. Since $a\left(b_{1 M}\right)=\underline{b}$ and $A$ is continuous, there must exist some $\bar{b} \in\left(b_{1 M}, \hat{b}\right)$ for which $a(\bar{b})=b_{1 M}$. Again, for such a choice, the DM must believe that all future asset choices must be $\underline{b}$. Given these beliefs, however, $h(\bar{b})$ is the optimal choice so $a(\bar{b})=h(\bar{b})$. But $h(b)<b_{1 M}$ for $b \in\left(b_{1 M}, b_{2 M}\right)$. Since $a(\bar{b})$ cannot be both equal to and less than $b_{1 M}$, supposition (ii) must also be false. As (i) and (ii) exhaust all possible cases, we conclude that there cannot exist any $\hat{b} \in\left(b_{1 M}, b_{2 M}\right)$ for which $a(\hat{b}) \neq h(\hat{b})$. Therefore, for any MPE with continuous $a(b), a(b)$ must satisfy the form stated in the Lemma for $b \in\left(b_{1 M}, b_{2 M}\right]$.

Having pinned down the form of the $a(b)$ function over $\left[\underline{b}, b_{2 M}\right]$ under the assumption of continuity, we can now show that the DM can do better by departing from it. Specifically, we will show that at $b=b_{2 M}$ the DM would strictly prefer to choose a $b^{\prime}>b_{1 M}$, whereas 
Lemma 1 asserts that for a continuous $a(b), a\left(b_{2 M}\right)=h\left(b_{2 M}\right)=b_{1 M}$.

Theorem 3 There is no MPE for which $a(b)$ is continuous for all $b$.

Proof. (By contradiction) Suppose, to get a contradiction, that a continuous $a(b)$ exists. Now, consider a DM with $b=b_{2 M}$ who is contemplating choosing $b^{\prime} \in\left[b_{1 M}, b_{2 M}\right]$. By Lemma 1 , the DM can predict what future DMs will choose for every $b^{\prime} \in\left[b_{1 M}, b_{2 M}\right]$. Using these predictions, this choice problem can be formulated as follows:

$$
\begin{aligned}
& \max _{b^{\prime}} U\left(y+b_{2 M}-\beta b^{\prime}\right)+ \\
& \delta\left[\beta U\left(y+b^{\prime}-\beta a\left(b^{\prime}\right)\right)+\beta^{2} U\left(y+a\left(b^{\prime}\right)-\beta \underline{b}\right)+\beta^{3} \frac{U(\bar{y}+(1-\beta) \underline{b})}{(1-\beta)}\right]
\end{aligned}
$$

s.t.

$$
b_{1 M} \leq b^{\prime} \leq b_{2 M}
$$

Observe that for $b^{\prime} \in\left[b_{1 M}, b_{2 M}\right], a\left(b^{\prime}\right) \in\left[\underline{b}, b_{1 M}\right]$ and, so, $a\left(a\left(b^{\prime}\right)\right)=\underline{b}$. We will show that the right-hand derivative of the objective function at $b^{\prime}=b_{1 M}$ is strictly positive. Note that the right-hand and left-hand derivative of $a(b)$ are different at $b_{1 M}$, but in this program we are only considering $b^{\prime}$ choices that are at least as large as $b_{1 M}$. The derivative of the objective function is:

$$
-U^{\prime}\left(y+b_{2 M}-\beta b^{\prime}\right)+\delta\left[U^{\prime}\left(y+b^{\prime}-\beta a\left(b^{\prime}\right)\right)\left(1-\beta a^{\prime}\left(b^{\prime}\right)\right)+\beta U^{\prime}\left(y+a\left(b^{\prime}\right)-\beta \underline{b}\right) a^{\prime}\left(b^{\prime}\right)\right]
$$

We know that $a^{\prime}\left(b^{\prime}\right)=\frac{\delta^{1 / \gamma}}{1+\delta^{1 / \gamma \beta}}$ for $b^{\prime} \in\left[b_{1 M}, b_{2 M}\right)$. So the FOC becomes:

$-U^{\prime}\left(y+b_{2 M}-\beta b^{\prime}\right)+\delta\left[U^{\prime}\left(y+b^{\prime}-\beta a\left(b^{\prime}\right)\right)\left(1-\frac{\beta \delta^{1 / \gamma}}{1+\delta^{1 / \gamma} \beta}\right)+\beta U^{\prime}\left(y+a\left(b^{\prime}\right)-\beta \underline{b}\right) \frac{\delta^{1 / \gamma}}{1+\delta^{1 / \gamma} \beta}\right]$

Evaluating the above at $b^{\prime}=b_{1 M}\left(\right.$ and recognizing that $a\left(b_{1 M}\right)=\underline{b}$ ) yields:

$-U^{\prime}\left(y+b_{2 M}-\beta b_{1 M}\right)+\delta\left[U^{\prime}\left(y+b_{1 M}-\beta \underline{b}\right)\left(1-\frac{\beta \delta^{1 / \gamma}}{1+\delta^{1 / \gamma} \beta}\right)+\beta U^{\prime}(y+\underline{b}-\beta \underline{b}) \frac{\delta^{1 / \gamma}}{1+\delta^{1 / \gamma} \beta}\right]$ 
By the definition of $b_{1 M}$ :

$$
U^{\prime}\left(y+b_{1 M}-\beta \underline{b}\right)=\delta U^{\prime}(y+\underline{b}-\beta \underline{b})
$$

Substituting the above into 15 gives:

$$
-U^{\prime}\left(y+b_{2 M}-\beta b_{1 M}\right)+U^{\prime}(y+\underline{b}-\beta \underline{b}) \delta\left(\frac{\delta+\beta \delta^{1 / \gamma}}{1+\delta^{1 / \gamma} \beta}\right)
$$

By the definition of $b_{2 M}$ :

$$
U^{\prime}\left(y+b_{2 M}-\beta b_{1 M}\right)=\delta^{2} U^{\prime}(y+\underline{b}-\beta \underline{b}) .
$$

But observe that since $\delta<1$,

$$
\delta<\frac{\delta+\beta \delta^{1 / \gamma}}{1+\delta^{1 / \gamma} \beta}
$$

Therefore, equation (17) implies that the expression in $(16)$ is strictly positive. Hence, the DM can obtain strictly higher utility with a $b^{\prime}>b_{1 M}$.

Note that the Theorem only establishes that $a(b)$ cannot be continuous everywhere. In particular, it does not establish that $a(b)$ must be discontinuous at $b_{2 M}$ since that result was derived under the assumption that $a(b)$ is continuous on the open interval $\left(b_{1 M}, b_{2 M}\right)$. The assumption may be false. Indeed, in the next section, we will provide conditions under which a MPE has a discontinuity in $\left(b_{1 M}, b_{2 M}\right)$.

An important implication of the lack of continuity of $a(b)$ is that $W(b)$ will also fail to be continuous. The reason is that the value function $V(b)$ has to be continuous, which, combined with the lack of continuity of $a(b)$ implies that $W(b)$ cannot be continuous everywhere. The next two theorems give the proof of continuity of $V(b)$ and the lack of continuity of $W(b)$. 
Theorem 4 In any MPE, $V(b)$ in (1) is strictly increasing and continuous in $b$.

Proof. The fact that $V(b)$ is strictly increasing in $b$ follows from the fact that a DM with higher $b$ can always replicate the choice of a DM with lower $b$ (so, continuation value will be the same) but obtain strictly higher current consumption ( $U$ is strictly increasing in $c$ ).

To prove that $V$ is continuous, suppose that $V(b)$ is not continuous at $\hat{b}$. We know from the monotonicity of $V$ that $\lim _{b \uparrow \widehat{b}} V(b)<V(\widehat{b})$ or $V(\widehat{b})<\lim _{b \sqrt{b}} V(b)$. Consider the first case. Since $c(\hat{b}) \geq \kappa>0$ (Corollary 2) for $b \in(\hat{b}-\kappa, \hat{b}), y+b-\beta a(\hat{b})>0$ and, so, $a(\hat{b})$ is feasible. Therefore, the DM can obtain $U(y+b-\beta a(\hat{b}))+\beta \delta W(a(\hat{b}))$. Since $U$ is continuous in $c, U(y+b-\beta a(\hat{b}))+\beta \delta W(a(\hat{b}))$ is continuous in $b \in(\hat{b}-\kappa, \hat{b})$. Since $V(b) \geq U(y+b-\beta a(\hat{b}))+\beta \delta W(a(\hat{b}))$ in this range, it follows that $\lim _{b \uparrow \widehat{b}} V(b) \geq V(\hat{b})$. Hence the first case is not possible.

Next consider the second case. Pick $\hat{b}+\mu, \mu<\kappa$. By Corollary 2, $c(\hat{b}+\mu) \geq \kappa$. Therefore, $c(\hat{b}+\mu)-\mu \geq \kappa-\mu>0$. Since $c(\hat{b}+\mu)-\mu=y+\hat{b}-\beta a(\hat{b}+\mu), a(\hat{b}+\mu)$ is feasible at $\hat{b}$ and delivers $U(y+\hat{b}-\beta a(\hat{b}+\mu))+\beta \delta W(a(\hat{b}+\mu))$. And, since $\lim _{\mu \rightarrow 0} U(y+\hat{b}-\beta(\hat{b}+\mu))+\beta \delta W(a(\hat{b}+\mu))=$ $\lim _{b \downarrow \widehat{b}} V(b)$ and $\lim _{b \downarrow \widehat{b}} V(b)>V(\widehat{b})$, there must exist some $\mu^{*}$ such that $a\left(\hat{b}+\mu^{*}\right)$ is feasible for $\hat{b}$ and provides more utility than the choice of $a(\hat{b})$. This contradicts the optimality of $a(\hat{b})$. Hence, the second case is not possible either. Therefore, $V(b)$ is continuous for all $b$.

Theorem 5 There is no MPE for which $W(b)$ is continuous for all $b$.

Proof. Let $\hat{b}$ be a point at which $a(b)$ is discontinuous. Select a sequence $\left\{b_{n}\right\}$ converging to $\hat{b}$ for which $\lim _{n} a\left(b_{n}\right)=a_{1} \neq a(\hat{b})$. By definition, $W\left(b_{n}\right)=U\left(y+b_{n}-\beta a\left(b_{n}\right)\right)+\beta W\left(a\left(b_{n}\right)\right)$ and $W(\hat{b})=U(y+\hat{b}-a(\hat{b}))+\beta W(a(\hat{b}))$.

Assume, to get a contradiction, that $W(b)$ is continuous. Then, $\lim _{n} W\left(b_{n}\right)=W(\hat{b})$ and $\lim _{n} W\left(a\left(b_{n}\right)\right)=W\left(a_{1}\right)$. Using the expression for $W\left(b_{n}\right)$ and $W(\hat{b})$ above and the fact that $U$ is continuous gives $U\left(y+\hat{b}-a_{1}\right)+\beta W\left(a_{1}\right)=U(y+\hat{b}-a(\hat{b}))+\beta W(a(\hat{b}))$, or,

$$
U\left(y+\hat{b}-a_{1}\right)-U(y+\hat{b}-a(\hat{b}))=\beta\left[W(a(\hat{b}))-W\left(a_{1}\right)\right] .
$$


Next, by continuity of $V$ and $U$ and the assumed continuity of $W, V(\hat{b})=\lim _{n} U\left(y+b_{n}-\right.$ $\left.\beta a\left(b_{n}\right)\right)+\delta \beta W\left(a\left(b_{n}\right)\right)=U\left(y+\hat{b}-\beta a_{1}\right)+\delta \beta W\left(a_{1}\right)$. Since $V(\hat{b})=U(y+\hat{b}-\beta a(\hat{b}))+\delta \beta W(a(\hat{b}))$, we obtain:

$$
U\left(y+\hat{b}-a_{1}\right)-U(y+\hat{b}-a(\hat{b}))=\delta \beta\left[W(a(\hat{b}))-W\left(a_{1}\right)\right]
$$

Since $a_{1} \neq a(\hat{b})$, both 18 and 19 cannot simultaneously be true for $\delta<1$. Hence $W(b)$ cannot be continuous for all $b$.

\subsection{Discontinuous Decision Rule and Multiple Steady States}

The fact that MPE decision rules are not continuous everywhere leads to a new channel through which debt levels other than $\underline{b}$ can be sustained as a steady state. This fact is important because it shows that the lack of continuity has substantive implications for the outcome of the model. Although the expectation is for dissaving behavior, dissaving need not happen at all points in equilibrium. This is not a mere theoretical curiosity because in the computations of this model, the finding of multiple steady states is the norm rather than the exception. The implications of multiple steady states are explored in the quantitative section.

We characterize one type of non-debt-limit steady state that might arise. To do so, it is convenient to define

$$
\begin{aligned}
& \Omega_{2}(b):[\underline{b}, \infty) \rightarrow \mathbb{R} \text { as: } \\
& \Omega_{2}(b)=\max _{b^{\prime}}\left\{U\left(y+b-\beta b^{\prime}\right)+\delta\left[\beta U\left(y+b^{\prime}-\beta \underline{b}\right)+\beta^{2} \frac{U(y+(1-\beta) \underline{b})}{(1-\beta)}\right]\right\} \\
& \text { s.t. } \\
& \underline{b} \leq b^{\prime} \leq b_{1 M}
\end{aligned}
$$


and $\Omega_{3}(b):[\underline{b}, \infty) \rightarrow R$ as:

$$
\begin{aligned}
& \Omega_{3}(b)=\max _{b^{\prime}} U\left(y+b-\beta b^{\prime}\right)+ \\
& \delta\left[\beta U\left(y+b^{\prime}-\beta h\left(b^{\prime}\right)\right)+\beta^{2} U\left(y+h\left(b^{\prime}\right)-\beta \underline{b}\right)+\beta^{3} \frac{U(y+(1-\beta) \underline{b})}{(1-\beta)}\right]
\end{aligned}
$$

s.t.

$$
b_{1 M} \leq b^{\prime} \leq b_{2 M} .
$$

Then, $\Omega_{2}(b)$ is the lifetime utility of the DM if he chooses $b^{\prime} \in\left[\underline{b}, b_{1 M}\right]$ and believes that the next period's DM will choose $\underline{b}$. Following our earlier notation (see (13)), the optimal decision rule for this problem is $h(b)$. And $\Omega_{3}(b)$ is the lifetime utility of the DM if he chooses $b^{\prime} \in\left[b_{1 M}, b_{2 M}\right]$ and believes that next period's DM will choose $b^{\prime}$ according to the function $h(b)$ and that the DM in the period after next will choose $\underline{b}$. Then, our candidate for a non-debt-limit steady state is a $b^{*} \in(\underline{b}, \infty)$ satisfying:

$$
\left(1+\frac{\beta \delta}{1-\beta}\right) U\left(y+(1-\beta) b^{*}\right)=\Omega_{2}\left(b^{*}\right) \geq \Omega_{3}\left(b^{*}\right) .
$$

The existence of such a $b^{*}$ is not guaranteed but if it exists then the following is true:

Theorem 6 If $b^{*} \in(\underline{b}, \infty)$ satisfying (20) exists then $b^{*} \in\left(b_{1 M}, b_{2 M}\right)$. If $a^{*}(b)$ is any MPE equilibrium of the restricted environment with $b \in\left[b^{*}, \infty\right)$, then

$$
a(b)= \begin{cases}h(b) & \text { for } b \in\left[\underline{b}, b^{*}\right) \\ b^{*} & \text { for } b=b^{*} \\ a^{*}(b) & \text { for } b \in\left(b^{*}, \infty\right)\end{cases}
$$

is a MPE of the environment with $b \in[\underline{b}, \infty)$.

Proof. See Appendix.

The proof of this proposition is somewhat lengthy and is given in the Appendix. Here we discuss several important points regarding it. First, the $h(b)$ function is the same function 
that we took as our guess for $a(b)$ under the assumption that $a(b)$ was continuous over the interval $\left[\underline{b}, b_{2 M}\right)$. Now, however, the assertion is that $a(b)$ is $h(b)$ over the smaller interval $\left[\underline{b}, b^{*}\right)$. And, since $h(b)<b_{1 M}$ for $b<b_{2 M}$ and $b^{*}>b_{1 M}, a(b)$ features an upward jump (discontinuity) at $b^{*}$. The discontinuity is the key as to why $b^{*}$ can be sustained as a steady state. The DM does not indulge its preference for current consumption because it knows that if it dissaved today, next period's DM will dissave substantially also. Since future DMs dissave too much from the current DM's perspective, this behavior is not desirable from current DM's perspective. Thus, the current DM weakly prefers to stay at $b^{*}$ rather than dissave.

Second, the Theorem asserts that any MPE equilibrium of the restricted environment where assets choices must lie in $\left[b^{*}, \infty\right)$ can be grafted on to the function specified in the Theorem for $b \in\left[\underline{b}, b^{*}\right]$ to constitute a MPE of the unrestricted environment. This is possible because, as shown in the Appendix, for $b \leq b^{*}$ the DM would prefer to choose $b^{*}$ over anything greater than $b^{*}$ and for $b>b^{*}$ the DM would prefer to choose $b^{*}$ over anything less than $b^{*}$.

Third, the existence of a non-debt-limit steady state opens up the possibility of many such steady states. For a DM with $b>b^{*}, b^{*}$ effectively serves as a hard debt limit. Suppose, then, that there exists $b^{* *}>b^{*}$ that satisfies a condition analogous to 20 with $b^{*}$ replaced by $b^{* *}$ and $\underline{b}$ replaced by $b^{*}$ in the definitions of $b_{1 M}, b_{2 M}, \Omega_{2}$ and $\Omega_{3}$. Then $b^{* *}$ can be supported as a steady state without altering the status of $b^{*}$ as a steady state. This process can (potentially) be carried out many times and explains why the finding of multiple steady states is common in computations (note that steady state $b$ 's need not be negative, i.e., the "debt limits" could simply be a lower bound on asset holdings).

\subsection{Discontinuous Decision Rule and Lotteries}

In this section we demonstrate that in the model presented thus far, the DM would strictly prefer to use lotteries at points where $a(b)$ is discontinuous. This demonstration serves as 
one motivation for developing the model with lotteries presented in the next section.

Theorem 7 Let $\{a(b), W(b)\}$ be a MPE. Then, lotteries dominate pure strategies at any point at which $a(b)$ is discontinuous

Proof. Let $\hat{b}$ be a point of discontinuity of $a(b)$. Select a sequence $\left\{b_{n}\right\}$ converging to $\hat{b}$ such that $\lim _{n} a\left(b_{n}\right)=\bar{a} \neq a(\hat{b})$. Note that since consumption is above $\kappa$ for all $b$, we can use the same argument as in Theorem 4 to establish that $\bar{a}$ is feasible at $\hat{b}$. Now consider the lottery, where $\bar{a}$ is chosen with probability $\lambda \in(0,1)$ and $a(\hat{b})$ with probability $(1-\lambda)$. The expected present value of this lottery is $\beta[\lambda \bar{a}+(1-\lambda) a(\hat{b})]$. If financial intermediaries are risk neutral, the lottery is feasible because $\bar{a}$ and $a(\hat{b})$ are both individually feasible at $\hat{b}$. The payoff to the DM from this lottery is $U(y+\hat{b}-\beta(\lambda \bar{a}+(1-\lambda) a(\hat{b})])+\delta \beta(\lambda W(\bar{a})+(1-\lambda) W(a(\hat{b}))]$. Since $V(b)$ is continuous in $b, \lim _{n} V\left(b_{n}\right)=U(y+\hat{b}-\beta \bar{a})+\delta \beta W(\bar{a})=V(\hat{b})$. Also, $V(\hat{b})=U(y+\hat{b}-$ $\beta a(\hat{b}))+\delta \beta W(a(\hat{b}))$. Therefore, each of the component pure strategies give the same payoff. Hence, for $\lambda \in(0,1), \lambda[U(y+\hat{b}-\beta \bar{a})+\delta \beta W(\bar{a})]+(1-\lambda)[U(y+\hat{b}-\beta a(\hat{b}))+\delta \beta W(a(\hat{b}))]=$ $U(y+\hat{b}-\beta a(\hat{b}))+\delta \beta W(a(\hat{b}))$. Now, observe that the l.h.s. of the preceding equality can be expressed as $\lambda U(y+\hat{b}-\beta \bar{a})+(1-\lambda) U(y+\hat{b}-\beta a(\hat{b}))+\delta \beta[\lambda W(\bar{a})+(1-\lambda) W(a(\hat{b}))]$. By strict concavity of $U, U(y+\hat{b}-\beta[\lambda \bar{a}+(1-\lambda) a(\hat{b})])>\lambda U(y+\hat{b}-\beta \bar{a})+(1-\lambda) U(y+\hat{b}-\beta a(\hat{b}))$. Therefore for $\lambda \in(0,1), U(y+\hat{b}-\beta[\lambda \bar{a}+(1-\lambda) a(\hat{b})])+\delta \beta[\lambda W(\bar{a})+(1-\lambda) W(a(\hat{b}))]>$ $U(y+\hat{b}-\beta a(\hat{b}))+\delta \beta W(a(\hat{b}))$. Hence, the lottery is strictly preferable to the (pure strategy) equilibrium decision.

The fact that lotteries help increase payoffs is an indication that continuation values are non concave. We can easily confirm this as follows. Since the pure strategy $b^{\prime}=\lambda \bar{a}+(1-$ $\lambda) a(\hat{b})$ is available to the DM but is not preferred to $a(\hat{b})$, it follows that $U(y+\hat{b}-\beta[\lambda \bar{a}+(1-$ $\lambda) a(\hat{b})])+\delta \beta W(\lambda \bar{a}+(1-\lambda) a(\hat{b})) \leq U(\bar{y}+\hat{b}-\beta a(\hat{b}))+\delta \beta W(a(\hat{b}))$. Since choosing $a(\hat{b})$ is strictly dominated by the lottery, it follows that $\lambda W(\bar{a})+(1-\lambda) W(a(\hat{b}))>W(\lambda \bar{a}+(1-\lambda) a(\hat{b}))$ for $\lambda \in(0,1)$. Thus $W(b)$ is strictly convex for $b$ between $\bar{a}$ and $a(\hat{b})$. This fact explains why lotteries are desirable: they allow the DM to replace any convex segment of $W(b)$ by that segment's concave upper envelope. 
We close this section with a comment on the difficulty of computing equilibria of the standard model. The issue is that the functions $a(b)$ and $W(b)$ cannot be accurately interpolated by standard techniques as both functions will have one or more points of discontinuity and the locations of these points are not known in advance. This means that even though $b$ is a continuous variable, it is awkward to treat it as such in the computations. On the other hand, if the space $[\underline{b}, \infty)$ is approximated by a grid then there is no assurance that the equations for $a(b)$ and $W(b)$ will have a solution on the grid. This computational awkwardness of the standard q-geometric model is a second motivation for developing the q-geometric discounting model with lotteries.

\section{Quasi-Geometric Discounting Model with Lotteries}

We extend the environment of the previous section to permit lotteries over next period's level of assets. Our goal in this section is to characterize equilibria for this lottery-enhanced environment, establish the existence of at least one equilibrium, and discuss the key steps in the computation of an equilibrium.

Both the proof of existence of an equilibrium and its computation requires that we restrict ourselves to a bounded choice set. Henceforth, we will assume that the set of possible asset choices belong to the closed interval $[\underline{b}, \bar{b}]$, where $\underline{b} \geq-y /(1-\beta)$. If $\underline{b}$ is equal to its lower bound, we require that $U$ be well-defined over $c \geq 0$. Otherwise, it is sufficient that $U$ be well-defined on $c>0$. In either case, we will assume that $U(c)$ is strictly increasing, strictly concave and differentiable on the relevant domain.

Let $\mathscr{B}$ denote the Borel $\sigma$-algebra generated by the set $[\underline{b}, \bar{b}] \subset \mathbb{R}$. Let $\phi$ denote a finite measure on the measurable space $([\underline{b}, \bar{b}], \mathscr{B})$ and let $\Phi$ denote the set of all such measures. Let $\mathscr{W}$ be the set of all bounded functions defined on $[\underline{b}, \bar{b}]$ that are measurable with respect to $\mathscr{B}$.

Given a continuation value function $W(b) \in \mathscr{W}$, the DM solves the following dynamic 
program:

$$
V(b)=\sup _{\phi \in \Phi} U\left(y+b-\beta \int b^{\prime} \phi\left(d b^{\prime}\right)\right)+\delta \beta \int W\left(b^{\prime}\right) \phi\left(d b^{\prime}\right)
$$

s.t.

$$
\int \phi\left(d b^{\prime}\right)=1 \text { and } y+b-\beta \int b^{\prime} \phi\left(d b^{\prime}\right) \geq 0
$$

Definition 3 A Markov Perfect Equilibrium with Lotteries is a pair $\left\{\phi\left(b^{\prime}, b\right), W(b)\right\} \in \Phi \times \mathscr{W}$ such that the (probability) measure $\phi\left(b^{\prime}, b\right)$ solves (21) given $W(b)$ and $W(b)$ solves:

$$
W(b)=U\left(y+b-\beta \int b^{\prime} \phi\left(d b^{\prime}, b\right)\right)+\beta \int W\left(b^{\prime}\right) \phi\left(d b^{\prime}, b\right) .
$$

The appearance of a probability measure in the above definition gives the impression that a MPE with lotteries is a challenging equilibrium object to characterize and compute. However, we can reformulate the optimization problem (and the definition of an MPE) in such a way that the equilibrium probability measure does not make an explicit appearance (but can be inferred once the equilibrium is found). To do this, we first study the problem of choosing the best lottery with a given expected value $B$. Namely,

$$
\begin{aligned}
& w(B ; W)=\sup _{\phi \in \Phi} \int W(b) \phi(d b) \\
& \text { s.t. } \\
& \int \phi(d b)=1 \text { and } \int b \phi(d b)=B \text {. }
\end{aligned}
$$

Since $W(b) \in \mathscr{W}$, it is integrable with respect to any $\phi \in \Phi$ and since $W(b)$ is bounded, the bound on $W(b)$ also bounds the objective function for any choice of $\phi$. Therefore, the set of values taken by the objective function as $\phi$ ranges over the feasible set possesses a least upper bound and $w(B ; W)$ is well-defined. It is not immediately obvious that a lottery attaining the supremum exists but, generally speaking, it does. This follows from the fact that for this class of problems it is generally sufficient to optimize over the space of (atomic) 
measures that assign positive mass to at most as many points as there are constraints - in this case, two 9 If the choice set is restricted to 2-point lotteries, it is easy to show that a lottery attaining the supremum exists.

In what follows, it is assumed that a lottery attaining the supremum always exists (the fact that it is a 2-point distribution is not assumed, although this fact is useful for visualizing the results). Then we have the following important characterization of $w(B ; W)$.

Lemma 2 For any $W \in \mathscr{W}, w(B ; W):[\underline{b}, \bar{b}] \rightarrow \mathbb{R}$ is the concave upper envelope of $W(b)$, i.e., $w(B ; W)$ is the least concave function that majorizes $W(b)$.

Proof. We will first prove the $w(B ; W)$ is concave. Consider $B_{1}, B_{2}$ both elements of $\left.\underline{b}, \bar{b}\right]$. Let $\phi_{k}, k=1,2$, be the lotteries that attain $w\left(B_{k} ; W\right)$. By convexity of the constraint set, the probability measure defined by the compound lottery $\lambda \phi_{1}+(1-\lambda) \phi_{2}$ is feasible for $\lambda B_{1}+(1-\lambda) B_{2}$, where $\lambda \in(0,1)$, and delivers $\lambda w\left(B_{1} ; W\right)+(1-\lambda) w\left(B_{2} ; W\right)$. Hence $w\left(\lambda B_{1}+(1-\lambda) B_{2} ; W\right) \geq \lambda w\left(B_{1} ; W\right)+(1-\lambda) w\left(B_{2} ; W\right)$.

To prove the second part we must show that if $g(b) \geq W(b)$ and $g(b)$ is concave then $g(B) \geq w(B ; W)$. Observe that replacing $W(b)$ with $g(b)$ in 23 gives $w(B ; g) \geq w(B ; W)$. Since $g(b)$ is concave, we must have $w(B ; g)=g(B)$. Therefore $g(B) \geq w(B ; W)$.

The optimization problem 21 can now be re-stated as follows:

$$
V(b)=\max _{B^{\prime}} U\left(y+b-\beta B^{\prime}\right)+\delta \beta w\left(B^{\prime} ; W\right)
$$

s.t.

$B^{\prime} \in[\underline{b}, \bar{b}]$ and $y+b-\beta B^{\prime} \geq 0$.

Then we have the following equivalent definition of a MPE with lotteries:

Definition 4 A Markov Perfect Equilibrium with Lotteries (MPEL) is a pair $\{A(b), W(b)\}$

\footnotetext{
${ }^{9}$ These results follow from the theory of semi-infinite linear programs. See, for instance, Glashof and Gustafson (1983) and Hornstein and Prescott (1993).
} 
such that $A(b)$ solves (24) and $W(b)$ solves

$$
W(b)=U(y+b-\beta A(b))+\beta w(A(b) ; W),
$$

where $w(\cdot ; W)$ is the concave upper envelope of $W(b)$ (equivalently, $w(\cdot ; W)$ solves (23)).

This definition parallels the definition of a MPE in the standard q-geometric discounting model. The main difference is that the continuation value is no longer $W(b)$ but its concave upper envelope $w(\cdot ; W)$. Lotteries are implicit in this replacement but otherwise do not make an appearance.

\subsection{Characterization of Equilibrium}

The fact that $w(\cdot ; W)$ is concave implies well-behaved decision rules for any admissible $W(b)$. This fact is the reason why the lottery-enhanced model is both conceptually clean and computationally facile.

Theorem 8 For any $W \in \mathscr{W}, A(b)$ and $c(b)$ are continuous and increasing in $b$ and $A(b)$ is Lipschitz with constant $1 / \beta$.

\section{Proof.}

Continuity: In (24), $B^{\prime}$ is being chosen from a convex set and the objective function is strictly concave in $B^{\prime}$ because $U$ is strictly concave in $c$ and $w$ is concave in $B^{\prime}$. It follows from the Theorem of the Maximum (Stokey and Lucas (1989), Theorem 3.6, p. 62) that $A(b)$ is continuous in $b$. The continuity of $c(b)$ follows from the continuity of $U$ and the continuity of $A(b)$.

Monotonicity: The proof that $A(b)$ is increasing in $b$ is essentially the same as the proof that $a(b)$ is increasing in $b$ given in Theorem 1 and is therefore not repeated here. To show that $c(b)$ is increasing in $b$, we exploit the fact that $w(b ; W)$ is concave. Concavity implies that the left-hand and right-hand derivatives of $w(B ; W)$ with respect to $B$, denoted $w_{-}^{\prime}(B ; W)$ and 
$w_{+}^{\prime}(B ; W)$ respectively, exist for all interior points and $w_{+}^{\prime}(B ; W) \leq w_{-}^{\prime}(B ; W)$. Furthermore, optimality of $A(b)$ implies,

$$
\delta w_{+}^{\prime}(A(b) ; W) \leq U^{\prime}(y+b-\beta A(b)) \leq \delta w_{-}^{\prime}(A(b) ; W) .
$$

Take $b_{1}<b_{2}$. If $A\left(b_{1}\right)=A\left(b_{2}\right)$ then the result is obvious. If $A\left(b_{2}\right)>A\left(b_{1}\right)$, then from (26) we have $U^{\prime}\left(y+b_{2}-\beta A\left(b_{2}\right)\right) \leq \delta w_{-}^{\prime}\left(A\left(b_{2}\right)\right)$ and $U^{\prime}\left(y+b_{1}-\beta A\left(b_{1}\right)\right) \geq \delta w_{+}^{\prime}\left(A\left(b_{1}\right)\right)$.

From the concavity of $w$, we have $w_{-}^{\prime}\left(A\left(b_{2}\right)\right) \leq w_{+}^{\prime}\left(A\left(b_{1}\right)\right)$. Hence $U^{\prime}\left(y+b_{2}-\beta A\left(b_{2}\right)\right) \leq$ $w_{-}^{\prime}\left(A\left(b_{2}\right) ; W\right) \leq w_{+}^{\prime}\left(A\left(b_{1}\right) ; W\right) \leq U^{\prime}\left(y+b_{1}-\beta A\left(b_{1}\right)\right)$ which implies that $c\left(b_{1}\right) \leq c\left(b_{2}\right)$.

Lipschitz: Since $c(b)$ is increasing in $b, y+b_{1}-\beta A\left(b_{1}\right) \geq y+b_{0}-\beta A\left(b_{0}\right)$ for $b_{1}>b_{0}$ and $y+b_{1}-$ $\beta A\left(b_{1}\right) \leq y+b_{0}-\beta A\left(b_{0}\right)$ for $b_{1}<b_{0}$. For the first case, this implies, $b_{1}-b_{0} \geq \beta\left[A\left(b_{1}\right)-A\left(b_{0}\right)\right]$. For the second case, this implies $b_{1}-b_{0} \leq \beta\left[A\left(b_{1}\right)-A\left(b_{0}\right)\right]$, or $-\left(b_{1}-b_{0}\right) \geq-\beta\left[A\left(b_{1}\right)-A\left(b_{0}\right)\right]$. Combining the two cases, we have $1 / \beta \geq\left|A\left(b_{1}\right)-A\left(b_{0}\right)\right| /\left|b_{1}-b_{0}\right|$.

The analog of Theorem 1 holds here as well. However, since the bound applies to $A(b)$, realized $b^{\prime}$ (given $\left.A(b)\right)$ may exceed $b$.

Theorem 9 In any $M P E L, A(b) \leq b$.

Proof. The steps to establish that $A(b) \leq b$ are essentially the same as in Theorem 1 . The only difference is that we need an upper bound for $w(A(b) ; W)$ instead of $W(A(b))$. As stated in Theorem 1, $\widetilde{W}(b)$ is the maximum utility that the DM can get with only pure strategies. Since $\widetilde{W}(b)$ is strictly concave in $b$, the maximum utility the DM can get if it chooses a lottery over $b^{\prime}$ with expected value of $b$ is also $\widetilde{W}(b)$. The remaining steps are then exactly the same as in Theorem 1 .

A steady state is now a debt level $b$ such that the DM chooses $b$ with probability 1 . Then, we have the analog of the Corollary 1.

Corollary $3 \underline{b}$ is a steady state of any MPEL. 
Proof. By Theorem $8, A(\underline{b}) \leq \underline{b}$. Since a lottery cannot put any mass on values of $b<\underline{b}$, it follows that the DM must choose $\underline{b}$ with probability 1 at $\underline{b}$.

The analog of Corollary (2) also holds.

Corollary 4 In any MPEL, $c(b) \geq y+(1-\beta) b \geq \kappa>0$.

One important difference between the standard model and the model with lotteries is that in any MPEL, $W(b)$ is both continuous and monotone (but not generally concave).

Theorem 10 In any MPEL, $W(b)$ is continuous and increasing in $b$.

Proof. The continuity of $W(b)$ follows from the continuity of $w(b ; W)$ (a concave function is continuous), the continuity of $c(b)$ and the continuity of $U$. To prove monotonicity, let $b_{1}<b_{2}$ and suppose, to get a contradiction, $W\left(b_{2}\right)<W\left(b_{1}\right)$. Since, $c\left(b_{1}\right) \leq c\left(b_{2}\right)$ and $W(b)=U(y+b-\beta A(b))+\beta w(A(b) ; W)$, it follows that $w\left(A\left(b_{2}\right) ; W\right)<w\left(A\left(b_{1}\right) ; W\right)$. Then $A\left(b_{1}\right) \neq A\left(b_{2}\right)$ and from monotonicity of $A$ it follows that $A\left(b_{2}\right)>A\left(b_{1}\right)$. But this implies that the DM with $b_{2}$ will be strictly better off if it chose $A\left(b_{1}\right)$ (which is feasible for it) since that would give it strictly higher consumption today and strictly higher continuation utility. This contradicts the optimality of $A(b)$. Therefore, $W(b)$ is increasing in $b$.

\subsection{Existence of Equilibrium}

In this section, we establish the existence of a $M P E L$. Let $\mathscr{C}$ be the space of continuous functions defined on $[\underline{b}, \bar{b}]$. Let $\|f\|=\sup _{\underline{b} \leq b \leq \bar{b}}|f(b)|$ be the norm on $\mathscr{C}$. Let $\mathscr{F}=\{f \in \mathscr{C}$ : $f(b) \in[\underline{b}, \bar{b}]\}$. Then $\mathscr{F}$ is nonempty, bounded, closed and convex subset of $\mathscr{C}$ and $\mathscr{C}$ is a complete normed vector (Banach) space.

Lemma 3 For every $A \in \mathscr{F}$, there exists a unique $W(b ; A) \in \mathscr{C}$ that solves $W(b ; A)=$ $U(y+b-\beta A(b))+\beta w(A(b) ; W(b ; A))$. Furthermore, $W(b ; A)$ is continuous in $A$. 
Proof. Fix $A \in \mathscr{F}$ and define the operator $T_{A}$ as:

$$
\left(T_{A} W\right)(b)=U(y+b-\beta A(b))+\beta w(A(b) ; W) .
$$

We claim that (i) $\left(T_{A} W\right)(b) \in \mathscr{C}$, (ii) $T_{A}$ is a contraction map, and (iii) $T_{A}$ is continuous in $A$. Given these claims, the first part of the Lemma follows from (i) and (ii) and an application of the Contraction Mapping Theorem (Stokey and Lucas (1989), Theorem 3.2, p. 50). The second part follows easily from (iii); for a proof see Hutson and Pym (1980), Theorem 4.3.6, pp. 117-118.

Claim (i): Since $W \in \mathscr{C}$ is continuous, it is measurable with respect to space $([\underline{b}, \bar{b}], \mathscr{B})$. Therefore $w(B, W)$ exists. By Lemma 2, $w(B ; W)$ is concave and therefore continuous in $B$. Since $A$ is continuous in $b$ and $U$ is continuous in $c$, it follows that $\left(T_{A} W\right)(b) \in \mathscr{C}$.

Claim (ii): We verify Blackwell's sufficiency conditions for a contraction map. Consider $\widehat{W}(b) \geq W(b)$, both elements of $\mathscr{C}$. Clearly, $w(B ; \widehat{W}) \geq w(B ; W)$. Since $A$ is fixed, it follows that $\left(T_{A} \widehat{W}\right)(b) \geq\left(T_{A} W\right)(b)$. Hence $T_{A} W$ is monotone in $W$. Next, consider $\widehat{W}(b)=W(b)+$ $\theta$. Then, $w(B ; \widehat{W})=w(B ; W)+\beta \theta$. Again, since $A$ is fixed, it follows that $\left(T_{A} \widehat{W}\right)(b)=$ $\left(T_{A} W\right)(b)+\beta \theta$. Therefore, $T_{A}$ is a contraction map with modulus $\beta$.

Claim (iii): Fix $W \in \mathscr{C}$. Let $A \in \mathscr{F}$ and let $\left\{A_{n}\right\} \subset \mathscr{F}$ be a sequence such that $\lim _{n} \| A_{n}-$ $A \|=0$. From the continuity of $U(c)$ and $w(B ; W)$, the sequence $\left(T_{A_{n}} W\right)(b)=U(y+b-$ $\left.\beta A_{n}\right)+\beta w\left(A_{n} ; W\right)$ converges point-wise to $\left(T_{A} W\right)(b)=U(y+b-\beta A)+\beta w(A ; W)$. We will now show that $\lim _{n}\left\|T_{A_{n}} W-T_{A} W\right\|=0$. Observe that for any $b \in[\underline{b}, \bar{b}]$

$$
\begin{aligned}
& \mid U\left(y+b-\beta A_{n}+\beta w\left(A_{n} ; W\right)-U(y+b-\beta A-\beta w(A ; W) \mid\right. \\
& \leq \mid U\left(y+b-\beta A_{n}-U\left(y+b-\beta A|+| \beta w\left(A_{n} ; W\right)-\beta w(A ; W) \mid\right.\right. \\
& \leq U^{\prime}(y+(1-\beta) \underline{b})\left|A_{n}-A\right|+w_{+}^{\prime}(\underline{b} ; W) \beta\left|A_{n}-A\right| \\
& \leq U^{\prime}(y+(1-\beta) \underline{b})\left\|A_{n}-A\right\|+w_{+}^{\prime}(\underline{b} ; W) \beta\left\|A_{n}-A\right\|
\end{aligned}
$$


The first inequality follows from the triangle inequality; the second from the fact that $U$ and $w$ are both concave and the lowest consumption level possible is $y+(1-\beta) \underline{b}$ and the lowest asset level possible is $\underline{b}$; and the third from the definition of the norm $\|\cdot\|$. To complete the proof observe that since $b$ was arbitrary, the chain of inequalities will continue to hold if the first term in the chain is replaced by $\sup _{b \in[\underline{b}, \bar{b}]} \mid U\left(y+b-\beta A_{n}+\right.$ $\beta w\left(A_{n} ; W\right)-U\left(y+b-\beta A-\beta w(A ; W) \mid\right.$. But this would then imply $\left\|T_{A_{n}} W-T_{A} W\right\| \leq$ $U^{\prime}(y+(1-\beta) \underline{b})\left\|A_{n}-A\right\|+w_{+}^{\prime}(\underline{b} ; W) \beta\left\|A_{n}-A\right\|$. Since $\lim _{n}\left\|A_{n}-A\right\|=0$, it follows that $\lim _{n}\left\|T_{A_{n}} W-T_{A} W\right\|=0$.

We can now establish:

Theorem 11 A Markov perfect equilibrium with lotteries exists.

Proof. Since $W(b ; A)$ solves the functional equation 27 for a given $A(b)$, the existence of a MPEL is equivalent to the existence of $A^{*} \in \mathscr{F}$ such that:

$$
A^{*}(b)=\underset{B^{\prime} \in[\underline{b}, \bar{b}]}{\operatorname{argmax}} U\left(y+b-\beta B^{\prime}\right)+\delta \beta w\left(B^{\prime} ; W\left(b ; A^{*}\right)\right) .
$$

For any given $A \in \mathscr{F}$, define the operator $H$ as

$$
(H A)(b)=\underset{B^{\prime} \in[\underline{b}, b]}{\operatorname{argmax}} U\left(y+b-\beta B^{\prime}\right)+\delta \beta w\left(B^{\prime} ; W(b ; A)\right) .
$$

We claim that (i) $H$ is continuous, (ii) $H(\mathscr{F}) \subset \mathscr{F}$ and (iii) the family $H(\mathscr{F})$ is equicontinuous. Recalling that $\mathscr{F}$ is a nonempty, bounded, closed and convex subset of $\mathscr{C}$, the existence of a solution to 28 follows from an application of the Schauder Fixed Point Theorem (Stokey and Lucas (1989), Theorem 17.4, page 520).

Claim (i): Let $A \in \mathscr{F}$ and let $\left\{A_{n}\right\} \subset \mathscr{F}$ be a sequence such that $\lim _{n}\left\|A_{n}-A\right\|=0$. We wish to show that $\lim _{n}\left\|\left(H A_{n}\right)-(H A)\right\|=0$. For this, it is sufficient to show that $\lim _{n}\left\|w\left(\cdot ; W_{n}\right)-w(\cdot ; W)\right\|=0$, where $W_{n} \equiv W\left(b ; A_{n}\right)$ and $W \equiv W(b ; A)$. Now, observe that by Lemma 3 , there exists $N_{\epsilon}$ such that for all $n>N_{\epsilon},\left\|W_{n}-W\right\|<\epsilon$. Fix $B \in[\underline{b}, \bar{b}]$ and let $\phi_{n}^{B}$ and $\phi^{B}$ be the probability measures that attain $w\left(B, W_{n}\right)$ and $w(B, W)$, respectively. 
Then,

$$
\begin{aligned}
& \int\left(W\left(b ; A_{n}\right)-W(b ; A)\right) \phi^{B}(d b) \leq w\left(B ; W_{n}\right)-w(B ; W) \leq \int\left(W\left(b ; A_{n}\right)-W(b ; A)\right) \phi_{n}^{B}(d b) \\
& \Rightarrow \\
& -\int\left\|W_{n}-W\right\| \phi^{B} \leq w\left(B ; W_{n}\right)-w(B ; W) \leq \int\left\|W_{n}-W\right\| \phi_{n}^{B} \\
& \Rightarrow \\
& -\left\|W_{n}-W\right\| \leq w\left(B ; W_{n}\right)-w(B ; W) \leq\left\|W_{n}-W\right\| \\
& \Rightarrow \\
& \left|w\left(B ; W_{n}\right)-w(B ; W)\right| \leq\left\|W_{n}-W\right\| .
\end{aligned}
$$

Since $B$ was arbitrary, we have $\sup _{B}\left|w\left(B ; W_{n}\right)-w(B ; W)\right|=\left\|w\left(\cdot ; W_{n}\right)-w(\cdot ; W)\right\| \leq$ $\left\|W_{n}-W\right\|<\epsilon$ for all $n>N_{\epsilon}$. Hence, $\lim _{n}\left\|w\left(\cdot ; W_{n}\right)-w(\cdot ; W)\right\|=0$.

Claims (ii) and (iii): Let $A \in \mathscr{F}$. By Lemma $3, W(b ; A) \in \mathscr{C}$. Since $\mathscr{C} \subset \mathscr{W}$, by Theorem 8. $(H A)(b)$ is continuous and, of course, $(H A)(b) \in[\underline{b}, \bar{b}]$. Therefore $H(\mathscr{F}) \subset \mathscr{F}$. $(H A)(b)$ is also Lipschitz with constant $1 / \beta$ and, so, $H(\mathscr{F})$ is an equicontinuous family.

\subsection{Computation of Equilibrium}

In this section we give a short description of the basic steps for computing the equilibrium of the model.

The model is computed via discretization. Let $\left\{b_{1}, b_{2}, \ldots, b_{N}\right\} \subset[\underline{b}, \bar{b}]$ be the chosen grid. Suppose that $W_{k}\left(b_{i}\right), i=1,2, \ldots, N$ is the $W$ function delivered by the $(k-1)$ st iteration. By Lemma 2, $w\left(B ; W_{k}\right)$ is the concave upper envelope of $\left\{W_{k}\left(b_{i}\right)\right\}_{i=1}^{N}$. If we view these points as $N$ pair of points $\left\{\left(b_{i}, W_{k}\left(b_{i}\right)\right)\right\}_{i=1}^{N}$ in the plane, the concave upper envelope of $\left\{W_{k}\left(b_{i}\right)\right\}_{i=1}^{N}$ is the upper convex hull of the $N$ pair of points. There are many algorithms that compute the convex hull of arbitrary set of points in the plane. We use Andrew (1979), which goes as follows.

Order $\left\{b_{i}, W_{k}\left(b_{i}\right)\right\}_{i=1}^{N}$ in ascending order by $b$. Let $P^{k} \subseteq\left\{b_{i}, W_{k}\left(b_{i}\right)\right\}_{i=1}^{N}$ denote the set of 
points that make up the upper convex hull. The elements of $P^{k}$ are also ordered in ascending order by $b$. The set $P^{k}$ is built up recursively. Suppose we are at the stage of the recursion where the last element of $P^{k}$ is $\left(b_{j}, W_{k}\left(b_{j}\right)\right)$. Consider the point $\left(b_{j+1}, W_{k}\left(b_{j+1}\right)\right)$. If the line segment connecting $\left(b_{j}, W_{k}\left(b_{j}\right)\right)$ to $\left(b_{j+1}, W_{k}\left(b_{j+1}\right)\right)$ does not make a clockwise turn relative to the line segment connecting the last two element of $P^{k}$, drop the last element of $P^{k}$. If after the drop $P^{k}$ contains at least two elements, compare the segment connecting the new last element of $P^{k}$ to $\left(b_{j+1}, W_{k}\left(b_{j+1}\right)\right)$ with the line segment connecting the new last two elements of $P^{k}$. If the former does not make a clockwise turn relative to the latter, again drop the (new) last element of $P^{k}$. Continue this way until either the line segment connecting the last element of $P^{k}$ to $\left(b_{j+1}, W_{k}\left(b_{j+1}\right)\right)$ does make a clockwise turn relative to the line segment connecting the last two element of $P^{k}$ or there is only one element left in $P^{k}$. At this point, include $\left(b_{j+1}, W_{k}\left(b_{j+1}\right)\right)$ in $P^{k}$. Proceed to $\left(b_{j+2}, W_{k}\left(b_{j+2}\right)\right)$ and repeat the steps above again. This process is started with $P^{k}=\left\{\left(b_{1}, W_{k}\left(b_{1}\right)\right),\left(b_{2}, W_{k}\left(b_{2}\right)\right)\right\}$ and carried on until $\left(b_{N}, W_{k}\left(b_{N}\right)\right)$ has been included in $P^{k}$. The resulting $P^{k}$ set is the upper convex hull of $\left\{b_{i}, W_{k}\left(b_{i}\right)\right\}_{i=1}^{N}$. It always contains $\left(b_{1}, W_{k}\left(b_{1}\right)\right)$ and $\left(b_{N}, W_{k}\left(b_{N}\right)\right)$.

Turning to the computation of $A\left(b ; W_{k}\right)$, suppose that $P^{k}$ has $2 \leq J \leq N$ elements. Denote these by $\left\{p_{j}, W_{k}\left(p_{j}\right)\right\}_{j=1}^{J}$. Let $s_{j}, j=1,2, \ldots, J-1$, be the slopes between points $j$ and $j+1$. By construction, $s_{1}>s_{2}>\ldots>s_{J-1} . A\left(b_{i}\right), i=1,2, \ldots, N$, is computed as follows.

1. Evaluate $U^{\prime}\left(y+b_{i}-\beta b_{1}\right)$ (the marginal utility of current consumption if $\left.B^{\prime}=b_{1}\right)$. If $U^{\prime}\left(y+b_{i}-\beta b_{1}\right) \geq \delta s_{1}$, set $A\left(b_{i} ; W_{k}\right)=b_{1}$ and stop.

2. Otherwise, continue from $j=2$ though $J-1$ until either one of the below conditions are satisfied.

(a) If $U^{\prime}\left(y+b_{i}-\beta b_{j}\right) \geq \delta s_{j-1}$ and $U^{\prime}\left(y+b_{i}-\beta b_{j-1}\right) \leq \delta s_{j-1}$, solve for $\hat{B} \in\left[p_{j-1}, p_{j}\right]$ such that $U^{\prime}\left(y+b_{i}-\beta \hat{B}\right)=\delta s_{j-1}$, set $A\left(b_{i} ; W_{k}\right)=\hat{B}$ and stop ${ }^{10}$

10 If $\hat{B} \in\left(p_{j-1}, p_{j}\right), A\left(b_{i} ; W_{k}\right)$ is being assigned a value that is "off the grid." What this means is that the DM is choosing the grid $p_{j-1}$ with probability $\left[p_{j}-\hat{B}\right] /\left[p_{j}-p_{j-1}\right]$ and the grid $p_{j}$ with probability 
(b) If $U^{\prime}\left(y+b_{i}-\beta b_{j}\right)<\delta s_{j-1}$ and $U^{\prime}\left(y+b_{i}-\beta b_{j}\right)>\delta s_{j}$, set $A\left(b_{i} ; W_{k}\right)=b_{j}$ and stop.

3. If the above conditions not satisfied for any $j<J$ then for $j=J$ check condition (a) above and if true set $A\left(b_{i} ; W_{k}\right)$ accordingly. If false, set $A\left(b_{i} ; W_{k}\right)=b_{J}$.

To complete the description of the computational steps we note that $W$ is updated inertially as $W_{k+1}(b)=(1-\zeta) W_{k}\left(b_{i}\right)+\zeta X_{k}\left(b_{i}\right), \zeta \in(0,1)$, where

$$
\begin{aligned}
& X_{k}\left(b_{i}\right)=U\left(y+b_{i}-\beta A\left(b_{i} ; W_{k}\right)\right) \\
& \quad+\beta \begin{cases}W_{k}\left(b_{1}\right) & \text { if } A\left(b_{i} ; W_{k}\right)=b_{1}, \\
W_{k}\left(p_{j-1}\right)+s_{j-1}\left(A\left(b_{i} ; W_{k}\right)-p_{j-1}\right) & \text { if } A\left(b_{i} ; W_{k}\right) \in\left(p_{j-1}, p_{j}\right],\end{cases}
\end{aligned}
$$

is the discrete version of the r.h.s. of 25). From an initial $W_{0}$, the algorithm loops over $W$ until $\max _{i}\left|X_{k}\left(b_{i}\right)-W_{k}\left(b_{i}\right)\right|$ has dropped below $10^{-6}$ for some $k$ (by this point, $\max _{i}\left|A\left(b_{i} ; W_{k}\right)-A\left(b_{i} ; X_{k}\right)\right|$ is generally well below $10^{-6}$ as well).

\section{Smooth Solutions, Debt Limits and Lotteries}

We compute and compare decision rules from three different models. The first model is a standard q-geometric model with logarithmic preferences and no debt limit for which a closed form solution is available. This solution features a differentiable (and therefore continuous) decision rule. The second model is the same model as the first one but with a debt limit imposed. Third model is the same as the second model but with lotteries permitted.

As shown in Krusell, Kuruscu, and Smith (2002), when $U(c)=\log (c)$ and the gross interest rate is $R$, then without any debt limit, the decision rule is:

$$
A=\left[\frac{R \delta \beta}{(1-\beta+\delta \beta)}-1\right]\left(\frac{R y}{R-1}\right)+\frac{R \delta \beta}{(1-\beta+\delta \beta)} b
$$

When $R=1 / \beta$, the coefficient on $b$ is positive but strictly less than 1 (provided both $\delta$

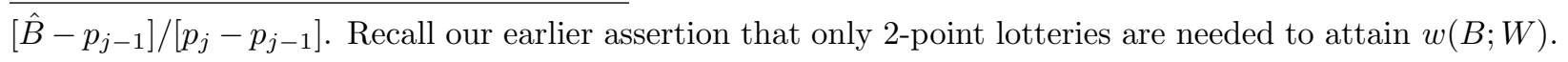


and $\beta$ are less than 1). Therefore, the solution implies that $b$ is declining over time. The implication for consumption is that it is declining over time and in the limit, converges to 0. In the limit, total debt converges to the negative of the present discounted value of the endowment stream.

Next, we consider the same model but assume that debt cannot exceed some lower bound $\underline{b} \leq 0$ that strictly exceeds the negative of the present discounted value of endowments. With this limit in place, there is no closed form solution available. To compute the model, we assume $y=1, \delta=0.98, R=1.05, \beta=1 / R$, and $\underline{b}=-0.25$. The computed decision rule is displayed in the top panel of Figure 1. The blue line is the 45-degree line and the green line is $a(b)$. Observe that there many jumps in the decision rule and also many steady states besides the one at $b=\underline{b} \cdot{ }^{11}$

In bottom panel of Figure 1, we plot the closed-form solution 29) along with the decision rules of the debt limit model with and without lotteries (the 45-degree line is omitted to reduce clutter). For the model with lotteries, we plot $A(b)$ which is the expected value of $b^{\prime}$ implied by the lottery. Observe that when $b$ is far away from the debt limit, the analytical solution and the solution from the model with lotteries are quite close and imply that the debt is increasing over time. In contrast, the behavior of the model with debt limit but without lotteries is quite different: there are many absorbing values of $b^{\prime}$ (corresponding to all the different non-debt limit steady states) which once attained is perpetuated forever. The panel also shows that there is only one absorbing state for the model with lotteries, namely, the debt limit.

One implication we wish to stress is that there is no way to recover the analytical solution (29) via standard finite-state value function iteration. The analytical solution requires that the debt limit be the negative of the present discounted value of earnings. If $\underline{b}$ is set to this limit, its choice would imply zero consumption and $V(\underline{b})=-\infty$. If we chose the debt limit to be very slightly greater than this so as to avoid unbounded negative payoffs, we are in

\footnotetext{
${ }^{11}$ An noted earlier, we are forced to treat $b$ as discrete variable with (possibly) attendant convergence issues. Fortunately, for our parameter values convergence was achieved.
} 
Figure 1
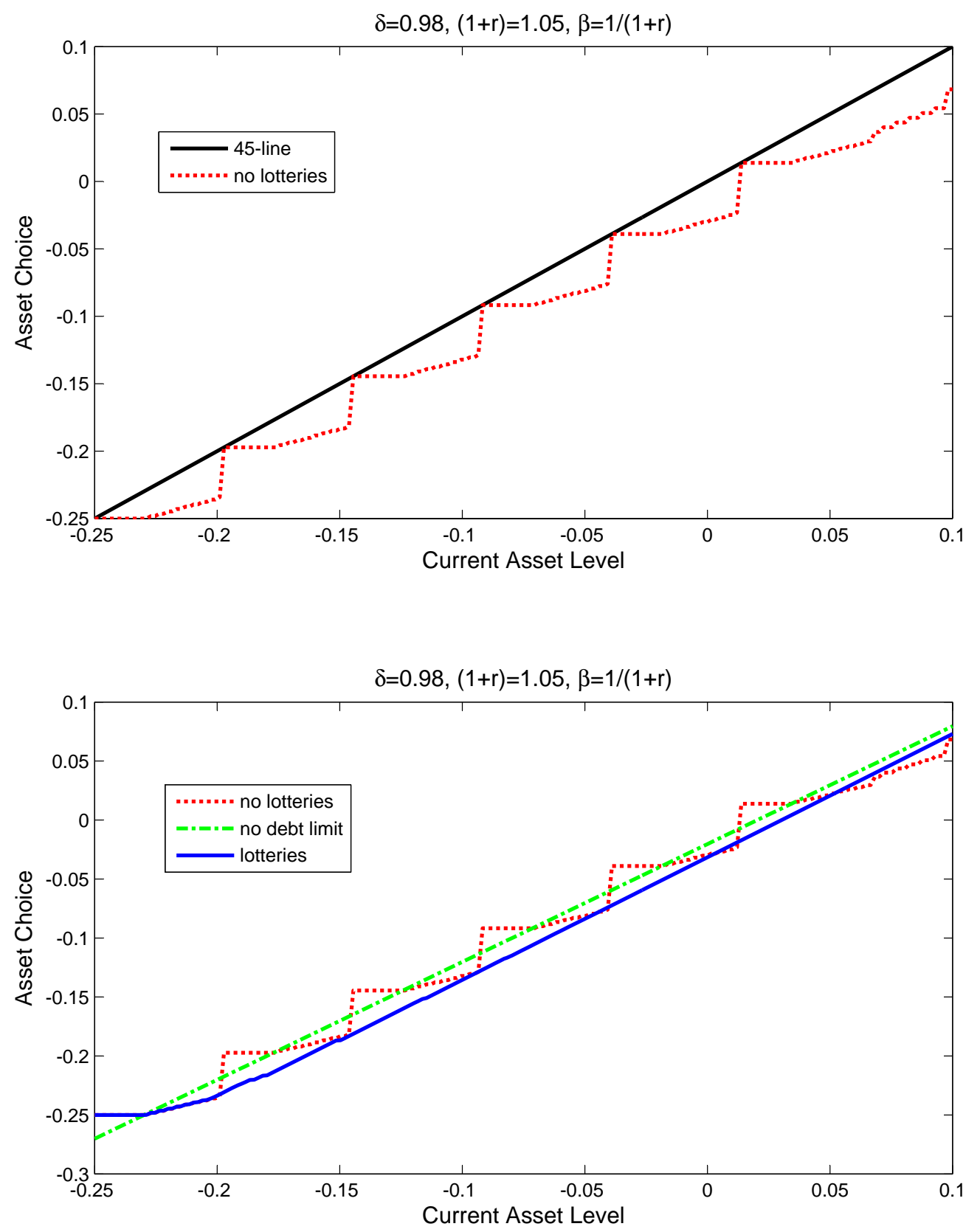
the realm of Theorem 3 and the model generates a discontinuous decision rule like the one shown in Figure 1. This result generalizes to any situation where there is a debt limit and the payoff from going to this limit is finite. This fact might explain why computing smooth solutions, in cases where an analytical solution is not known, often proves to be challenging. Basically, if the computational method implicitly imposes a lower bound on asset holdings that can actually be chosen, a smooth solution will not exist.

\section{Environment with Lotteries and Shocks}

In this section we extend the model with lotteries to include shocks to endowments. There are two motivations for this. First, for some applications the assumption of certainty is too restrictive and so, it may be worthwhile to see how uncertainty can be incorporated in the model with lotteries. Second, earlier work has suggested that uncertainty can play a role similar to that of lotteries in models of q-geometric discounting. Harris and Laibson (2001) have shown, for a problem similar to ours with a debt limit of zero, that MPE decision rules are continuous provided there is some uncertainty in endowments and $\delta$ is sufficiently close to 1 . This suggests that examining a model with both uncertainty and lotteries may be instructive.

Let $F(z, y), y, z \in Y \subset \mathbb{R}$ denote the CDF of next period's endowment $z$ given current period's endowment $y$. Let $W_{y}(b)$ denote the expected lifetime utility of a DM with assets $b$ at the start of next period, conditional on current period endowment being $y$. Then,

$$
\begin{aligned}
& w\left(B^{\prime} ; W_{y}\right)=\sup _{\phi \in \Phi} \int W_{y}(b) \phi(d b) \\
& \text { s.t. } \\
& \int \phi(d b)=1 \text { and } \int b \phi(d b)=B^{\prime},
\end{aligned}
$$




$$
A_{y}(b)=\underset{B^{\prime}}{\operatorname{argmax}} U\left(y+b-\beta B^{\prime}\right)+\delta \beta w\left(B^{\prime} ; W_{y}\right)
$$

s.t.

$$
B^{\prime} \in[\underline{b}, \bar{b}] \text { and } y+b-\beta B^{\prime} \geq 0
$$

and

$$
W_{y}(b)=\int\left[U\left(z+b-\beta A_{z}(b)\right)+\beta w\left(A_{z}(b) ; W_{z}\right)\right] F(d z, y)
$$

An MPEL is a collection of pairs $\left\{A_{y}(b), W_{y}(b)\right\}, y \in Y$, that satisfy $(30)-32$.

As is evident, the main modification is that $W(b)$ is replaced by $W_{y}(b)$ for each $y$ in the first-stage optimization problem (30) 12 If $Y$ is a discrete set, the computational steps for solving the model remain essentially the same as the model without shocks, except that the program now loops over the finite collection $\left\{W_{y}(b)\right\}_{y \in Y}$ and the optimization problem (31) is solved for each $y \in Y$. The proof of existence can be straightforwardly modified to handle the discrete $Y$ case.

In the case where endowment shocks are i.i.d., the model simplifies substantially. In this case we may treat $x=y+b$ as the state variable and solve for $W(x)$ and $A(x)$ (or $a(x)$ in the standard model). Except for the step that involves taking expectations over future $y$, the model is almost identical to the perfect certainty case.

\subsection{Numerical Comparision of the Standard Model and the Model with Lotteries}

We display the computed decision rules with and without lotteries in Figure 2, The parameter values for $\beta, R$ and $\delta$ are ones used in the previous section. The top panel reproduces the decision rules for the model with and without lotteries displayed earlier for $y=1$. The

\footnotetext{
${ }^{12}$ The implicit assumption is that the outcome of the lottery is independent of the realization of endowments.
} 


\section{Figure 2}
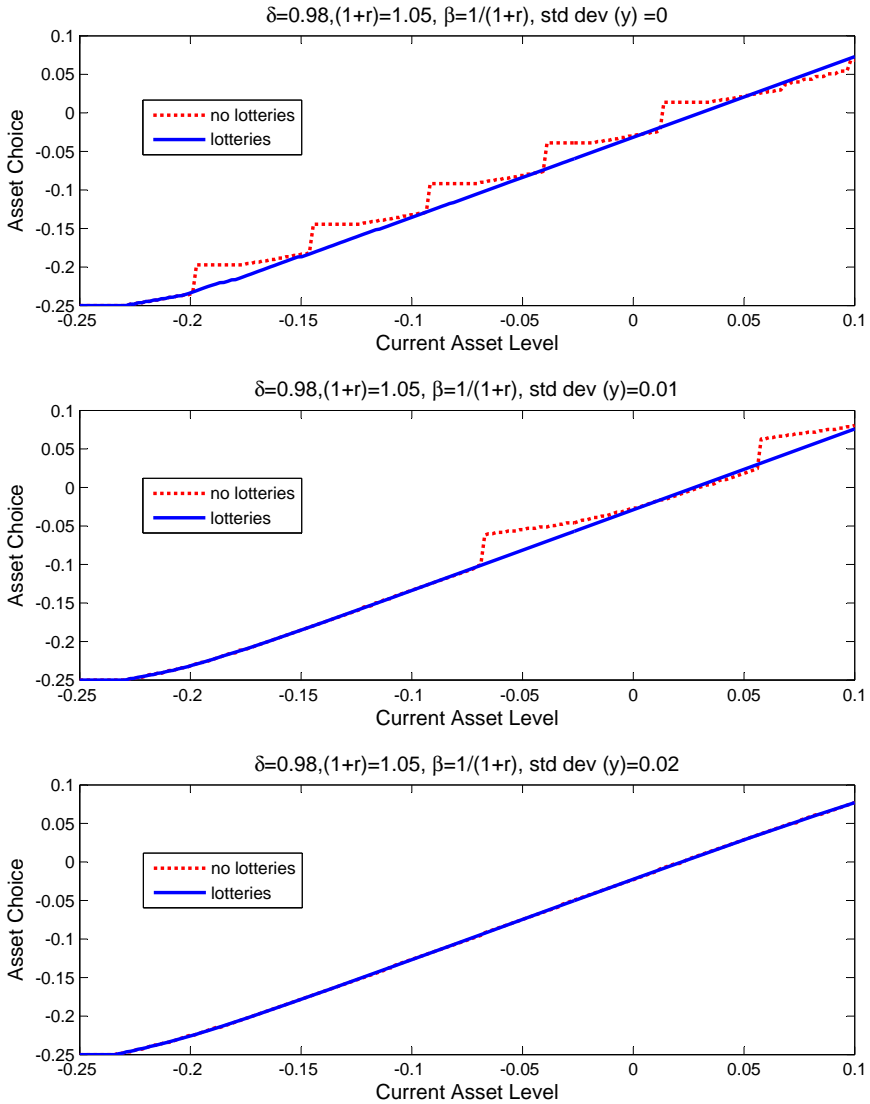
Figure 3
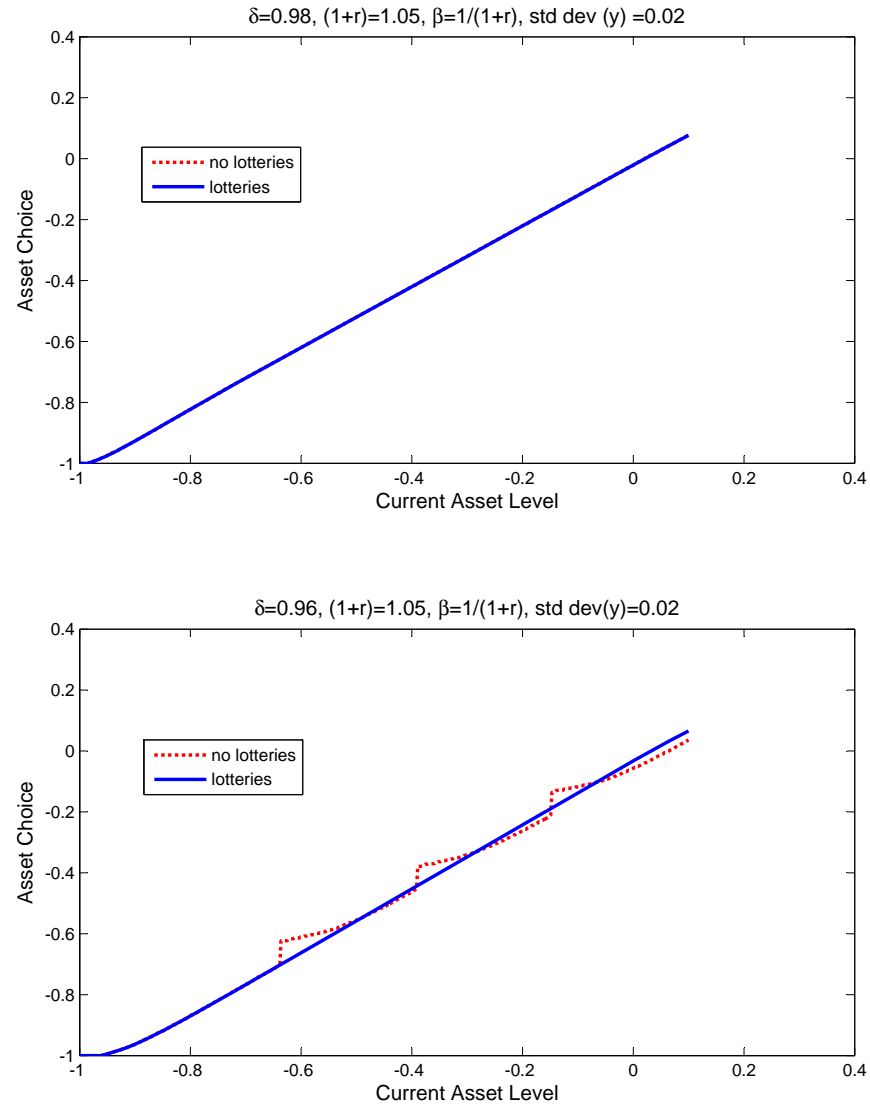
other two panels feature i.i.d. endowment shocks and for these we display the decision rules for $y=1$ (its mean value). In the middle panel, the standard deviation of shocks is 1 percent. The decision rule without lotteries continues to display jumps, although the frequency of jumps is less than in the perfect certainty case. Also, the two decision rules virtually coincide for a range of debt values. The bottom panel displays the case where the standard deviation of shocks is raised to 2 percent. Now, the jumps in decision rule for standard model disappear altogether and the decision rules in the two models are seemingly identical.

These results confirm that sufficient randomness in (future) endowments can "smooth out" decision rules. The fact that the decision rules from the standard model and the model with lotteries are almost identical is informative about why this is the case. Evidently, uncertainty has the effect of making continuation values almost concave. If continuation values are almost concave, adding lotteries should make very little difference to the equilibrium decision rules and that is what we find 13

Lest the above findings leave the reader with the impression that shocks are a good substitute for lotteries, we report what happens if the endowment volatility is held constant but the value of $\delta$ is lowered slightly. We fix the standard deviation of endowment shocks at 2 percent but increase the degree of present bias by lowering $\delta$ from 0.98 to 0.96 . For this exercise, we changed the debt limit to $\underline{b}=-1.00$. The results are displayed in Figure 3 which are plotted, again, for the mean value of $y=1$. The top panel displays the decision rule for $\delta=0.98$. As is evident, the decision rule for the standard model does not display any jumps and is seemingly identical to the decision rule for the model with lotteries. The bottom panel shows how decision rules change as $\delta$ is lowered slightly to 0.96 . Now, the decision rule in the

\footnotetext{
${ }^{13}$ That being said, there is an important difference in the robustness of computations across the two models. As mentioned earlier, when the standard model is solved on a grid there is no assurance that a solution exists on the grid. In fact, for our choices of standard deviation of $y$ (of 1 and 2 percent), the standard models fails to converge even though it converges for the perfect certainty case. In Figure 2 we simply display the decision rule for the standard model after the value function has been iterated 3000 times. In contrast, for the model with lotteries, convergence of both value and policy functions is attained within a tolerance of $10^{-6}$ for the same number of iterations, regardless of whether the standard deviation of $y$ is 0,1 or 2 percent.
} 

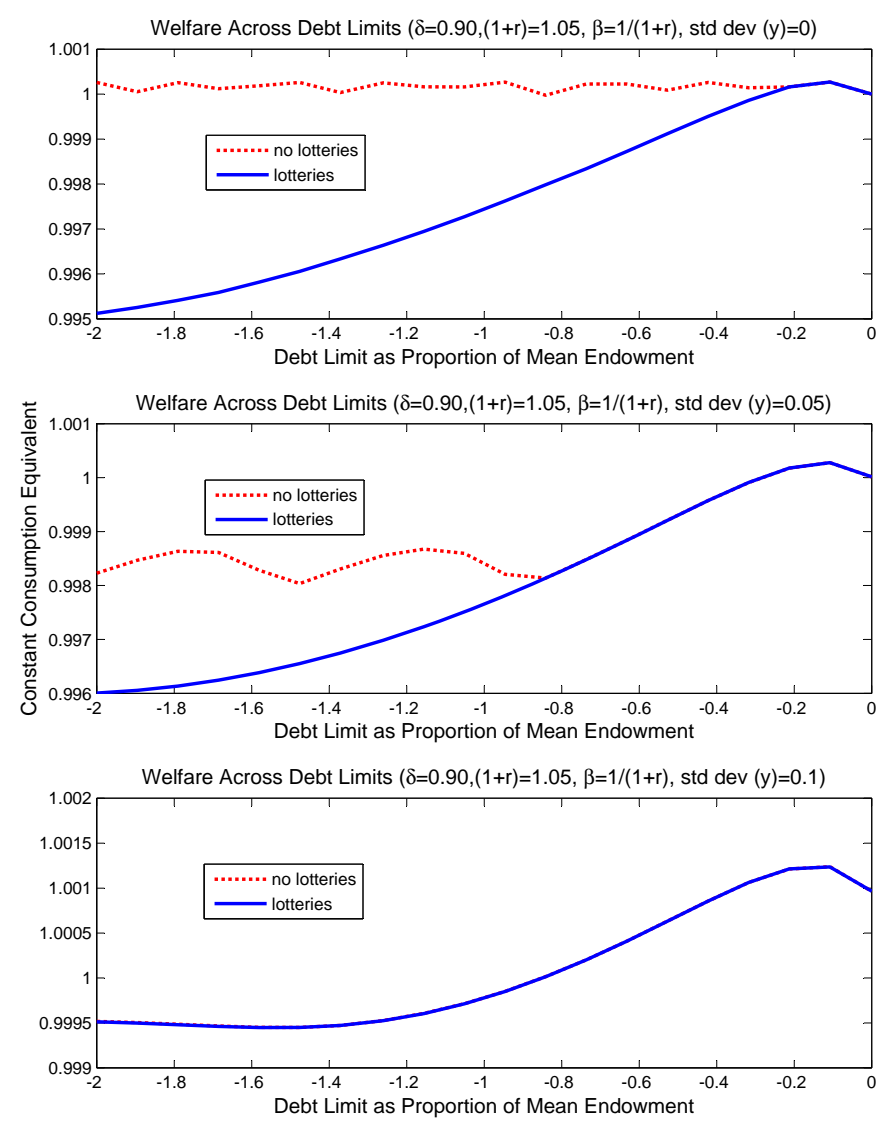

standard model is no longer continuous. Thus, even a rather small change in present bias can reintroduce significant non-concavities in continuation values and consequent jumps in decision rules.

\section{Welfare of the Current DM and the Debt Limit}

In this section we explore the implications of discontinuity of decision rules in the standard model for a substantive policy issue. In our environment, a relevant policy question is how welfare of the current DM varies with the debt limit. Whether we are concerned with bankruptcy regimes for individuals or for countries, there are many policies whose ultimate effect is to alter debt limits. We show that the discontinuities in decision rules in the standard q-geometric model can have unappealing implications for how welfare of the current DM 
varies with the debt limit.

In the top panel of Figure 4, we show how the welfare of the current DM, who starts with zero debt, varies with $\underline{b}$ when endowments are constant over time. For the model with lotteries, welfare initially increases with $\underline{b}$ but then declines. This accords well with intuition. In the absence of any present bias $(\delta=1)$, the DM is indifferent between different debt limits since it prefers to maintain a constant consumption over time. However, the fact that $\delta<1$ means the DM would prefer to consume more in the current period than in all future periods. This can be accomplished by a $\underline{b}$ that is exactly large enough to support the additional consumption desired in the initial period. As $\underline{b}$ is increased beyond this ideal level, welfare increasingly declines because DMs end up consuming too much in current and near future and too little in the long run (when the debt limit is reached).

For the model without lotteries, however, the relationship between debt limit and welfare is not as intuitive. As in the model with lotteries, welfare begins to decline as $\underline{b}$ is increased beyond the ideal debt limit but then it begins to rise again. The reason for this odd behavior is that $\underline{b}$ affects the location of the first non-debt-limit steady state to the left of zero. In particular, as the debt limit expands, the location of this steady state can move closer to the ideal debt limit or further away from it. Since the first non-steady-state debt limit is where the DM ends up in the long run, welfare can rise and fall with an expansion of $\underline{b}$.

Since a non-debt-limit steady state can be sustained over time only if there are no shocks, this behavior of welfare may seem specific to the perfect certainty case. However, the middle panel in Figure 4 shows that the relationship between welfare and $\underline{b}$ continues to feature undulations over some range of $\underline{b}$ even when there is substantial volatility in endowments $(5$ percent) 14

The reason can be understood from the decision rules displayed in Figure 5. The decision rules are plotted for 3 different levels of $y$. All decision rules feature discontinuities at roughly similar debt levels. For the medium and high output levels, these discontinuities are preceded

\footnotetext{
${ }^{14}$ Although these undulations occur well past the ideal debt limit, their presence can still be important if low debt limits are infeasible for some other reason.
} 
Figure 5

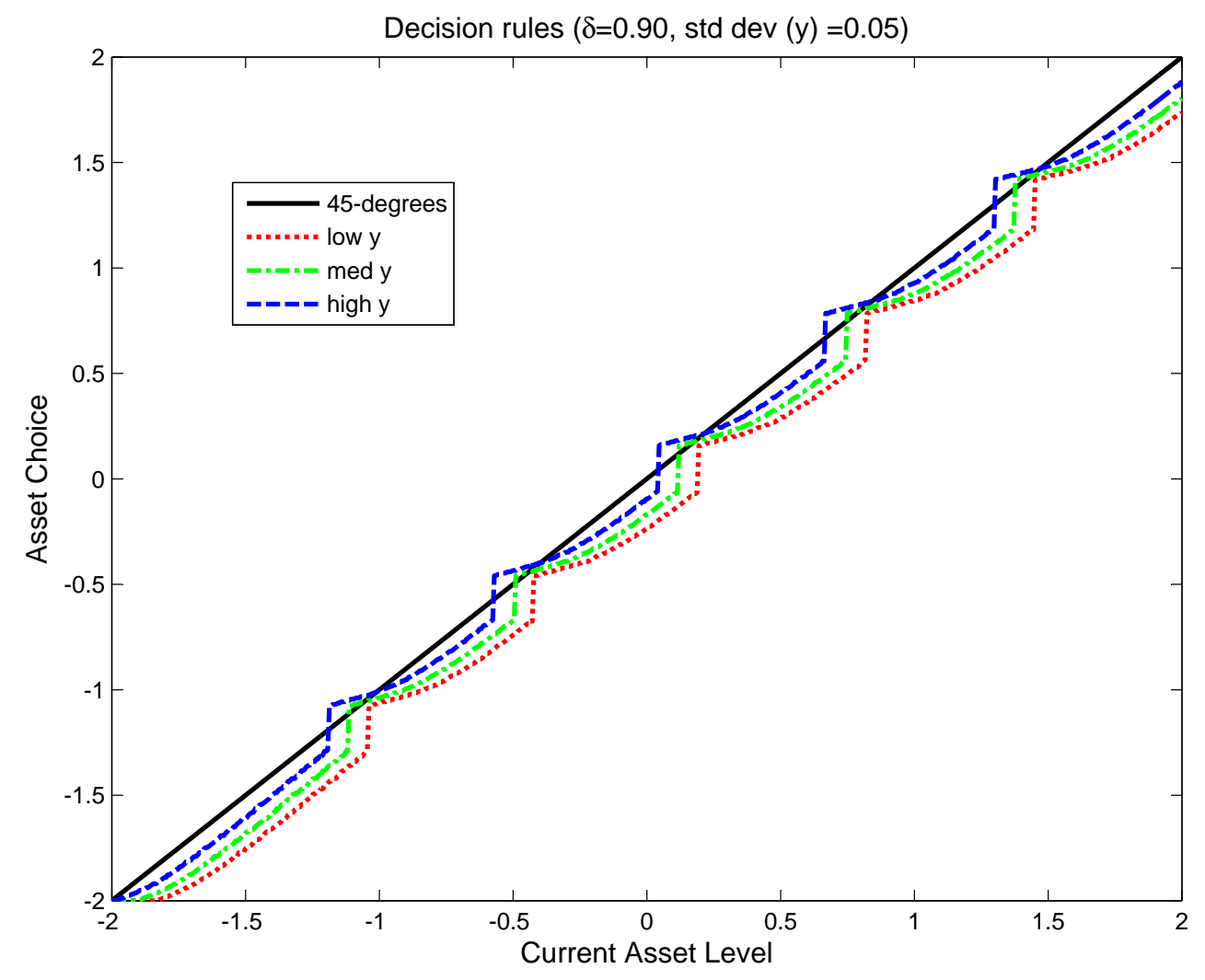


by relatively flat segments that cross the 45 -degree line. This means that there is a range of endowment shocks for which the $b^{\prime}$ stays within some neighborhood of these intersection points. Thus, these points act like "attractors" around which the debt levels can fluctuate for some length of time until there is a bad shock that abruptly pushes the DM toward a much higher debt level. The fact that debt levels can (endogenously) spend significant amount of time around these "attractors" makes their location important to the calculation of welfare. And, as in the perfect certainty case, the location of these "attractors" can move closer to or further away from the ideal debt limit as $\underline{b}$ is varied.

Finally, we note that if the standard deviation of endowment shocks is raised to 10 percent, the relationship between welfare and $\underline{b}$ takes on the hump-shaped form of the model with lotteries. With enough volatility, decision rules in the standard model is virtually the same as in the model with lotteries and the behavior of welfare with the respect to $\underline{b}$ is also the same. This is shown in the bottom panel of Figure 4 .

\section{Conclusion}

Models with quasi-geometric discounting are known to be challenging to analyze and compute. In this paper we dug deeper into why these complications arise and suggested a reformulation of the standard quasi-geometric choice problem using lotteries. The reformulation has the twin benefits of logical coherence (existence of an MPE is assured) and computational ease. Importantly, the behavior of the model with lotteries accords well with our economic intuition: We expect an intertemporal choice problem with present bias to imply dissaving behavior until such dissaving is arrested by a debt limit (or by rising interest rates, more generally). With lotteries permitted, this is a robust prediction the model. 


\section{References}

Acemoglu, D., M. Golosov, and A. Tsyvinski (2011): "Power Fluctuations and Political Economy," Journal of Economic Theory, 146, 1009-1041.

Aguiar, M., and M. Amador (2011): "Growth in the Shadow of Expropriation," Quarterly Journal of Economics, 126, 651-697.

Alesina, A., and G. Tabellini (1990): "A Positive Theory of Fiscal Deficits and Government Debt in a Democracy," Review of Economic Studies, 57, 403-414.

Andrew, A. (1979): "Another Efficient Algorithm for Convex Hulls in Two Dimensions," Information Processing Letters, 9, 216-219.

Azzimonti, M. (2011): "Barriers to Investment in Polarized Societies," American Economic Review, 101(5), 2182-2204.

BArro, R. J. (1999): "Ramsey Meets Laibson in the Neoclassical Growth Model," Quarterly Journal of Economics, 114(4), 1125-1152.

Battagliani, M., and S. Coate (2008): "A Dynamic Theory of Public Spending, Taxation and Debt," American Economic Review, 98, 201-236.

Glashof, K., And S.-A. Gustafson (1983): Linear Optimization and Approximation. Springer-Verlag.

Harris, C., And D. Laibson (2001): "Dynamic Choices of Hyperbolic Consumers," Econometrica, 69(4), 935-957.

HertzBerg, A. (2012): "Exponential Individuals, Hyperbolic Households," Unpublished.

Hornstein, A., and E. C. Prescott (1993): "The Firm and the Plant in General Equilibrium Theory," in General Equilibrium, Growth and Trade II: The Legacy of Lionel McKenzie, ed. by R. Becker, M. Boldrin, R. Jones, and W. Thomson, pp. 393-410. Academic Press. 
Hutson, V., And J. S. Pym (1980): Applications of Functional Analysis and Operator Theory. Academic Press, NY, USA.

JudD, K. L. (2004): "Existence, Uniqueness, and Computational Theory for Time Consistent Equilibria: A Hyperbolic Discounting Example," unpublished, Hoover Institution.

Krusell, P., B. Kuruscu, and A. A. Smith (2002): "Equilibrium Welfare and Government Policy with Quasi-Geometric Discounting," Journal of Economic Theory, 105, $42-72$.

Krusell, P., and A. A. Smith (2003): "Consumption-Savings Decisions with QuasiGeometric Discounting," Econometrica, 71(1), 365-375.

Laibson, D. (1996): "Hyperbolic Discount Functions, Undersaving, and Savings Policy," National Bureau of Economic Research, (Working Paper No. 5636).

- (1997): "Golden Egss and Hyperbolic Discounting," Quarterly Journal of Economics, pp. 443-477.

Laibson, D., A. Repetto, and J. Tobacman (1998): "Self-control and Saving for Retirement," Brookings Papers on Economic Activity, 1, 91-196.

Maliar, L., and S. Maliar (2005): "Solving the Neoclassical Growth Model with QuasiGeometric Discounting: A Grid-Based Euler-Equation Method," Computational Economics, 26, 163-172.

Peleg, B., And M. E. YaAri (1973): "On the Existence of a Consistent Course of Action when Tastes are Changing," Review of Economic Studies, 40(3), 391-401.

Persson, T., And L. Svensson (1989): "Why a Stubborn Conservative Would Run a Deficit: Policy with Time-Inconsistent Preferences," Quarterly Journal of Economics, $104,325-345$. 
Phelps, E. S., and R. A. Pollak (1968): "On Second-Best National Saving and GameEquilibrium Growth," Review of Economic Studies, 35(2), 185-199.

Stokey, N. L., and R. E. Lucas (1989): Recursive Methods in Economic Dynamics. Harvard University Press, MA, USA.

\section{A Equivalence Between the Q-Geometric Discounting and Political Disagreement Models}

There are two political parties, denoted by $N$ and $S$, representing two types of people of equal measure. At any date, one of the types is in power and constitutes the government. Types change power randomly over time, with the probability that the type (or party) currently in power remains in power next period with probability $1 / 2$. The level of revenues available each period is $y$.

Governments might spend both on their own constituents and the opposing party's constituents. Let $\left(g_{N}, g_{S}\right)$ denote the spending on the two types of constituents. The utility obtained by the government of each type is:

$$
u_{N}\left(g_{N}, g_{S}\right)=\frac{g_{N}^{1-\gamma}}{1-\gamma}+\theta \frac{g_{S}^{1-\gamma}}{1-\gamma} \text { and } u_{S}\left(g_{N}, g_{S}\right)=\theta \frac{g_{N}^{1-\gamma}}{1-\gamma}+\frac{g_{S}^{1-\gamma}}{1-\gamma},
$$

where $\theta \in[0,1]$ and $0<\gamma<1$. The parameter $\theta$ captures the degree of polarization in the economy: If $\theta=0$ then neither type of government gets any benefit from expenditure directed toward the other type's constituents; if $\theta=1$ then both types care equally about the expenditure directed toward the other type. The parameter $\gamma$ controls how rapidly the marginal benefit of government expenditure declines with spending.

Let us suppose that region $k \in\{N, S\}$ is in power and optimally allocates available resources between the two types. Given the utility functions, it follows that $g_{\sim k}=\theta^{1 / \gamma} g_{k}$. 
Since nothing depends on the identity of the party in power, we need only distinguish governments by whether they are in power or not. Let $g$ denote the per-capita spending on members of party in power. Then, the period utility of party in power is:

$$
u_{P}(g)=\varphi_{P} \frac{g^{1-\gamma}}{1-\gamma}, \quad \text { where } \varphi_{P}=1+\theta^{1 / \gamma}
$$

and the period utility of the party that is out of power, as function of $g$, is:

$$
u_{O}(g)=\varphi_{O} \frac{g^{1-\gamma}}{1-\gamma}, \quad \text { where } \varphi_{O}=\theta+\theta^{\frac{1-\gamma}{\gamma}}
$$

Since $\theta \in[0,1]$ and $1 \geq \gamma>0, \varphi_{P} \geq \varphi_{O}$, where the equality holds only if $\theta=1$ or $\gamma=1$.

We can express the decision problem of the party in power recursively as follows:

$$
V_{P}(b)=\max _{b^{\prime} \in B} \varphi_{P} \frac{g^{1-\gamma}}{1-\gamma}+\beta\left[0.5 *\left(V_{P}\left(b^{\prime}\right)+V_{O}\left(b^{\prime}\right)\right)\right]
$$

s.t.

$$
g=\frac{1}{p}\left[y+b-q b^{\prime}\right] \geq 0
$$

where $p=1+\theta^{1 / \gamma}$ and $q$ is the price of the bond. Let $a(b)$ denote the solution to this decision problem. Then, the life-time utility of the party that is currently out of power is:

$$
\begin{aligned}
V_{O}(y, b) & =\varphi_{O} \frac{(g(b))^{1-\gamma}}{1-\gamma}+\beta\left[0.5 *\left(V_{P}(a(b))+V_{O}(a(b))\right)\right] . \\
g(b) & =\frac{1}{p}[y+b-q a(b)]
\end{aligned}
$$


Now observe that

$$
\begin{aligned}
& \left(V_{P}(b)+V_{O}(b)\right)=\left(\varphi_{P}+\varphi_{O}\right) \frac{(g(b))^{1-\gamma}}{1-\gamma}+\left[\left(V_{P}(a(b))+V_{O}(a(b))\right)\right] \\
& \Rightarrow \\
& \frac{\left(V_{P}(b)+V_{O}(b)\right)}{\left(\varphi_{P}+\varphi_{O}\right)}=\frac{g(b)^{1-\gamma}}{1-\gamma}+\beta\left[\frac{\left.\left(V_{P}(a(b))\right)+V_{O}(a(b))\right)}{\left(\varphi_{P}+\varphi_{O}\right)}\right]
\end{aligned}
$$

Define

$$
W(b)=\frac{V_{P}(b)+V_{O}(b)}{\varphi_{P}+\varphi_{O}} \text { and } V(b)=\left(1 / \varphi_{P}\right) V_{P}(b) .
$$

Then,

$$
W(b)=\frac{g(b)^{1-\gamma}}{1-\gamma}+\beta W(a(b))
$$

and

$$
V(b)=\max _{b^{\prime} \in B} \frac{g^{1-\gamma}}{1-\gamma}+\beta \frac{0.5\left(\varphi_{P}+\varphi_{O}\right)}{\varphi_{P}} W\left(b^{\prime}\right)
$$

s.t.

$g=\frac{1}{p}\left[y+b-q b^{\prime}\right] \geq 0$

Aside from the normalizing factor $1 / p,(33)-(34)$ are identical to the key equations of the quasi-geometric discounting model in the text, with $\delta=0.5\left(\varphi_{P}+\varphi_{O}\right) / \varphi_{P} \leq 1$ and $q=\beta$.

\section{B Proof of Theorem 6}

Lemma $4 \Omega_{3}^{\prime}(b)-\Omega_{2}^{\prime}(b) \geq 0$.

Proof. Denote the optimal decision rule for $\Omega_{3}(b)$ as $a_{3}(b)$ and the optimal decision rule for $\Omega_{2}(b)$ as $h(b)$. Then $\Omega_{3}^{\prime}(b)=U^{\prime}\left(y+b-\beta a_{3}(b)\right)$. When the FOC is satisfied with an equality, 
the above follows from the envelope theorem. If the FOC holds with an inequality, it follows from the fact that $a_{3}(b)$ does not change with $b$. Similarly, $\Omega_{2}^{\prime}(b)=U^{\prime}(y+b-\beta h(b))$. By the definition of the choice sets $a_{3}(b) \geq h(b)$ and, hence, $\Omega_{3}^{\prime}(b)-\Omega_{2}^{\prime}(b) \geq 0$.

We can now give the proof of the first part of Theorem 6 .

Part 1: If $b^{*}$ exists, $b^{*} \in\left(b_{1 M}, b_{2 M}\right)$.

Proof. To show $b^{*}<b_{2 M}$, we will first show that $\Omega_{3}\left(b_{2 M}\right)-\Omega_{2}\left(b_{2 M}\right)>0$. Note that $\Omega_{2}\left(b_{2 M}\right)$ is the value of the program (13) with $b=b_{2 M}$ and $\Omega_{3}\left(b_{2 M}\right)$ is the value of the program in (14) with $b=b_{2 M}$. By Theorem $3, \Omega_{3}\left(b_{2 M}\right)-\Omega_{2}\left(b_{2 M}\right)>0$. Since $\Omega_{3}^{\prime}(b)-\Omega_{2}^{\prime}(b) \geq 0$ it follows that if $b^{*}$ exists, $b^{*}<b_{2 M}$ in order for $\Omega_{2}\left(b^{*}\right) \geq \Omega_{3}\left(b^{*}\right)$.

To show that $b^{*}>b_{1 M}$, we show that $\left(1+\frac{\beta \delta}{1-\beta}\right) U(y+(1-\beta) b)<\Omega_{2}(b)$ for $b \in\left(\underline{b}, b_{1 M}\right]$ which implies that the equality condition in (20) cannot be satisfied in this range. Consider the following problem for $b \in\left(\underline{b}, b_{1 M}\right]$ :

$$
\Phi(b)=\max _{b^{\prime} \geq \underline{b}} U\left(y+b-\beta b^{\prime}\right)+\delta \beta\left[\frac{U\left(y+(1-\beta) b^{\prime}\right)}{(1-\beta)}\right] .
$$

Then $\Phi(b)=\Omega_{2}(b)$ since in both problems the optimal choice is $\underline{b}$ and at that choice the two values are the same. Next, observe that in program in $(35), b^{\prime}=b$ was available but not chosen. It follows from strict concavity of $U$ that $\Omega_{2}(b)>\left(1+\frac{\beta \delta}{1-\beta}\right) U(y+(1-\beta) b)$ for $b \in\left(\underline{b}, b_{1 M}\right]$. Therefore, if $b^{*}>\underline{b}$ exists, $b^{*}$ must strictly exceed $b_{1 M}$.

To establish the second part of Theorem 6, we need two additional Lemmas.

Lemma 5 Let $\Omega_{3}^{R}(b)$ be the value of the program that defines $\Omega_{3}(b)$ with the choice set restricted to $\left[b_{1 M}, b^{*}\right)$. Then, for $b \in\left(b_{1 M}, b^{*}\right], \Omega_{3}^{R}(b)=\Omega_{3}(b)$.

Proof. The key here is that $a_{3}(b)$ is constrained at its lower bound until some $\tilde{b}>b_{1 M}$ and beyond that point $a_{3}(b)$ rises at a slope less than 1 . Therefore regardless of whether $\tilde{b}$ is to the left or right of $b^{*}$, the optimal choice in $\Omega_{3}(b)$ for $b \in\left[b_{1 M}, b^{*}\right]$ is strictly less than $b^{*}$.

Let $\tilde{b}$ be the value of $b$ for which $a_{3}(b)=b_{1 M}$ and the constraint that $b^{\prime} \geq b_{1 M}$ just ceases to bind. In this case, the FOC condition for optimality of $a_{3}(b)$ holds with an equality and 
gives:

$$
-U^{\prime}\left(y+\tilde{b}-\beta b_{1 M}\right)+U^{\prime}(y+\underline{b}-\beta \underline{b})\left(\delta \frac{\delta+\beta \delta^{1 / \gamma}}{1+\beta \delta^{1 / \gamma}}\right)=0
$$

Next, we claim that the l.h.s of 36 is strictly less than 0 at $\tilde{b}=b_{1 M}$. From definition of $b_{1 M}$ we have that that first term on the l.h.s. of $(36)$, after we substitute $b_{1 M}$ for $\tilde{b}$, is:

$$
U^{\prime}\left(y+b_{1 M}-\beta b_{1 M}\right)=\left(\frac{\left(1-\beta+\beta \delta^{1 / \sigma}\right)}{\delta^{1 / \sigma}}\right)^{-\sigma} U^{\prime}(y+\underline{b}-\beta \underline{b}) .
$$

Since

$$
\left(1-\beta+\beta \delta^{1 / \sigma}\right)^{-\sigma}>1>\left[\delta+\beta \delta^{1 / \sigma}\right] /\left[1+\beta \delta^{1 / \sigma}\right]
$$

the 1.h.s. of 36 is negative for $\tilde{b}=b_{1 M}$. Now observe that from concavity of $U$, the l.h.s. of 36 is strictly increasing in $\tilde{b}$ and, hence, the expression will be 0 for some $\tilde{b}>b_{1 M}$.

Next, note that in the region where the upper and lower constraints on $b^{\prime}$ do not bind

$$
a_{3}^{\prime}(b)=\frac{\left(\delta \frac{\delta+\beta \delta^{1 / \sigma}}{1+\beta \delta^{1 / \sigma}}\right)^{1 / \sigma}}{\left[\frac{\delta^{1 / \sigma}}{1+\delta^{1 / \sigma} \beta}+\beta\left(\delta \frac{\delta+\beta \delta^{1 / \sigma}}{1+\beta \delta^{1 / \sigma}}\right)^{1 / \sigma}\right]}
$$

which we can verify is strictly less than 1 .

Hence $\Omega_{3}^{R}(b)=\Omega_{3}(b)$ for $b \in\left(b_{1 M}, b^{*}\right]$.

Lemma 6 Let $\Omega_{2}^{R}(b)$ be the value of the program that defines $\Omega_{2}(b)$ with the choice set restricted to $b^{\prime} \in\left[\underline{b}, b_{1 M}\right)$. Then, for $b \in\left[\underline{b}, b^{*}\right], \Omega_{2}^{R}(b)=\Omega_{2}(b)$. 
Proof. Observe that:

$$
h(b)= \begin{cases}\underline{b} & b \in\left[\underline{b}, b_{1 M}\right] \\ \frac{\left(-\left(1-\delta^{1 / \gamma}\right) y+\delta^{1 / \gamma} b+\beta \underline{b}\right)}{1+\delta^{1 / \gamma \beta}} & b \in\left(b_{1 M}, b_{2 M}\right) \\ b_{1 M} & b \in\left[b_{2 M}, \infty\right)\end{cases}
$$

From the above we see that $h(b)<b_{1 M}$ for all $b<b_{2 M}$. Since $b^{*}<b_{2 M}$, the restriction of $b^{\prime}$ to $\left[\underline{b}, b^{*}\right]$ is not binding for $b \in\left[\underline{b}, b^{*}\right]$. Therefore, $\Omega_{2}^{R}(b)=\Omega_{2}(b)$.

We are now ready to give the proof of the second part of Theorem 6 .

Part 2: If $a^{*}(b)$ is any MPE equilibrium of the environment with $b \in\left[b^{*}, \infty\right)$, then

$$
a(b)= \begin{cases}h(b) & \text { for } b \in\left[\underline{b}, b^{*}\right) \\ b^{*} & \text { for } b=b^{*} \\ a^{*}(b) & \text { for } b \in\left(b^{*}, \infty\right)\end{cases}
$$

is a MPE of the environment with $b \in[\underline{b}, \infty)$

\section{Proof.}

For $b \in\left[\underline{b}, b_{1 M}\right], a(b)=h(b)=\underline{b}$ in any MPE by Corollary 1 and Theorem 2 .

For $b \in\left(b_{1 M}, b^{*}\right)$, we need to show that the DM prefers $b^{\prime}=h(b)$. We show this by considering four mutually exclusive alternatives: (i) $b^{\prime} \in\left[\underline{b}, b_{1 M}\right.$ ), which would imply payoff $\Omega_{2}^{R}(b)$, (ii) $b^{\prime} \in\left[b_{1 M}, b^{*}\right)$ which would imply payoff $\Omega_{3}^{R}(b)$, (iii) $b^{\prime}=b^{*}$, and (iv) $b^{\prime}>b^{*}$. First, we show that (i) dominates (ii). Lemmas 4 , 5 and 6 imply $\Omega_{3}^{R \prime}(b)-\Omega_{2}^{R^{\prime}}(b)=\Omega_{3}^{\prime}(b)-\Omega_{2}^{\prime}(b) \geq 0$. By (20) $\Omega_{2}\left(b^{*}\right) \geq \Omega_{3}\left(b^{*}\right)$. Therefore, $\Omega_{3}^{R}(b)<\Omega_{2}^{R}(b)$ for $b \in\left(b_{1 M}, b^{*}\right)$. Second, we show that (i) dominates (iii). For this, define $\Omega_{b^{*}}(b)=U\left(y+b-\beta b^{*}\right)+\frac{\beta \delta}{1-\beta} U\left(y+(1-\beta) b^{*}\right)$. We know that $\Omega_{b^{*}}^{\prime}(b)=U^{\prime}\left(y+b-\beta b^{*}\right)$ and $\Omega_{2}^{\prime}(b)=U^{\prime}(y+b-\beta h(b))$. For the region $b \in\left[b_{1 M}, b^{*}\right), h(b)<$ $b^{*}$ and, so, $\Omega_{b^{*}}^{\prime}(b)>\Omega_{2}^{\prime}(b)$. By $(20), \Omega_{b^{*}}\left(b^{*}\right)=\Omega_{2}\left(b^{*}\right)$ so $\Omega_{b^{*}}(b)<\Omega_{2}(b)$ for $b \in\left[b_{1 M}, b^{*}\right)$. Finally, we show that (iii) dominates (iv). By an argument similar to that given in Theorem 2. we may show that for all $b \leq b^{*}, U\left(y+b-\beta b^{*}\right)+\delta \beta \widetilde{W}\left(b^{*}\right)>U\left(y+b-\beta b^{\prime}\right)+\delta \beta \widetilde{W}\left(b^{\prime}\right)$ for $b^{\prime}>b^{*}$. Since $b^{*}$ is a steady state, $U\left(y+b-\beta b^{*}\right)+\delta \beta W\left(b^{*}\right)>U\left(y+b-\beta b^{\prime}\right)+\delta \beta W\left(b^{\prime}\right)$ 
for all $b^{\prime}>b^{*}$. Hence, $h(b)$ is the optimal choice for $b \in\left(b_{1 M}, b^{*}\right)$.

For $b=b^{*}$, we showed above that the DM will never choose $b^{\prime}>b^{*}$. If it chooses something less than $b^{*}$, the best it can do is given by $\Omega_{2}^{R}\left(b^{*}\right)$ which is the same as $\Omega_{2}\left(b^{*}\right)$ by Lemma 6 (as $\left.\Omega_{2}\left(b^{*}\right) \geq \Omega_{3}\left(b^{*}\right)\right)$. And, by (20) we know that $\Omega_{2}\left(b^{*}\right)=\Omega_{b^{*}}\left(b^{*}\right)$. Therefore the DM is indifferent between choosing $b^{*}$ or something less.

For $b \in\left(b^{*}, \infty\right)$, we need to show that it is still optimal to choose $a^{*}(b)$. Since $b^{*}$ is a steady state of the restricted as well as the unrestricted environments (and therefore has the same continuation values in both environments) and $a^{*}$ is a MPE of the restricted environment, it is sufficient to show that a DM with $b>b^{*}$ will never choose $b^{\prime}<b^{*}$ in which case $a^{*}(b)$ will continue to describe the equilibrium strategies for the unrestricted environment. At $b=b^{*}, U\left(y+b-\beta b^{*}\right)+\delta \beta W\left(b^{*}\right) \geq U\left(y+b-\beta b^{\prime}\right)+\delta \beta W\left(b^{\prime}\right)$ for all $b^{\prime}<b^{*}$. Differentiating with respect to $b$ gives $U^{\prime}\left(y+b-\beta b^{*}\right)>U^{\prime}\left(y+b-\beta b^{\prime}\right)$. Therefore for all $b>b^{*}, U\left(y+b-\beta b^{*}\right)+\delta \beta W\left(b^{*}\right)>U\left(y+b-\beta b^{\prime}\right)+\delta \beta W\left(b^{\prime}\right)$ for $b^{\prime}<b^{*}$. 